\title{
Autonomous Synchronization of Chemically Coupled Synthetic Oscillators
}

\section{Journal Article}

\section{Author(s):}

Lang, Moritz; Marquez-Lago, Tatiana T.; Stelling, Jörg; Waldherr, Steffen

Publication date:

2011

Permanent link:

https://doi.org/10.3929/ethz-b-000040425

\section{Rights / license:}

In Copyright - Non-Commercial Use Permitted

Originally published in:

Bulletin of Mathematical Biology 73(11), https://doi.org/10.1007/s11538-011-9642-8 


\title{
Autonomous Synchronization of Chemically Coupled Synthetic Oscillators
}

\author{
Moritz Lang • Tatiana T. Marquez-Lago • \\ Jörg Stelling • Steffen Waldherr
}

Received: 9 March 2010 / Accepted: 4 February 2011 / Published online: 4 March 2011

(C) Society for Mathematical Biology 2011

\begin{abstract}
Synthetic biology has recently provided functional single-cell oscillators. With a few exceptions, however, synchronization across a population has not been achieved yet. In particular, designing a cell coupling mechanism to achieve autonomous synchronization is not straightforward since there are usually several different design alternatives. Here, we propose a method to mathematically predict autonomous synchronization properties, and to identify the network structure with the best performance, thus increasing the feasibility for a successful implementation in vivo.

Our method relies on the reduction of ODE-based models for synthetic oscillators to a phase description, and the subsequent analysis of the phase model either in the spatially homogeneous or heterogeneous case. This analysis identifies three major factors determining if and when autonomous synchronization can be achieved, namely cell density, cell to cell variability, and structural design decisions. More-
\end{abstract}

Electronic supplementary material The online version of this article

(doi:10.1007/s11538-011-9642-8) contains supplementary material, which is available to authorized users.

M. Lang (凶) · T.T. Marquez-Lago $(\bowtie) \cdot$ J. Stelling

Department of Biosystems Science and Engineering, and Swiss Institute of Bioinformatics, ETH

Zürich, Mattenstrasse 26, 4058 Basel, Switzerland

e-mail: moritz.lang@bsse.ethz.ch

url: http://www.csb.ethz.ch

T.T. Marquez-Lago $(\bowtie)$

e-mail: tatiana.marquezlago@gmail.com

J. Stelling

e-mail: joerg.stelling@bsse.ethz.ch

S. Waldherr

Institute for Systems Theory and Automatic Control, University of Stuttgart, Pfaffenwaldring 9,

70550 Stuttgart, Germany

e-mail: waldherr@ist.uni-stuttgart.de

url: http://www.ist.uni-stuttgart.de 
over, when considering a spatially heterogeneous medium, we observe phase waves. These waves may hinder synchronization substantially, and their suppression should be considered in the design process.

In contrast to previous work, we analyze the synchronization process of models of experimentally validated synthetic oscillators in mammalian cells. Alternative designs for cell-to-cell communication via a quorum sensing mechanism differ in few mechanistic details, but these differences have important implications for autonomous synchronization. Our analysis suggests that not only the periodical transcription of the protein producing the signaling molecule, but also of the receptor protein is necessary to achieve good performance.

Keywords Synchronization · Synthetic oscillators · Kuramoto analysis · Spatial waves

\section{Introduction}

Biomolecular clocks can be found in many types of cells. In multicellular organisms, oscillators residing in individual cells often synchronize their signals with those of neighboring cells. This requires some communication mechanism between the cells, upon which they are able to produce a common oscillatory signal, to which each cell subsequently synchronizes.

In the last decade several synthetic oscillators were constructed that mimic properties of natural oscillatory networks (Elowitz and Leibler 2000; Atkinson et al. 2003; Fung et al. 2008; Stricker et al. 2008; Tigges et al. 2009). These synthetic oscillators have helped broadening our understanding of the intrinsic dynamics of natural oscillators, due to their relative "simplicity."

In principle, it is possible to synchronize synthetic single-cell oscillators, and a synthetic oscillator with the ability of autonomous synchronization was recently engineered in Escherichia coli (Danino et al. 2010). By using a microfluidic device, the authors showed the dependencies between the synchronization process and changing flow conditions. However, the rational design of systems that allow synchronization of entire cell populations remains a challenge, as much as prediction mechanism assessing the synchronization properties of alternative designs.

Previous theoretical works in the field often focused on abstract or simplified oscillator models, for instance, to compare attractive, repulsive, and stochastic coupling of populations of relaxation oscillators, smooth oscillators, and stochastic oscillators (Zhou et al. 2008). These studies typically rely on numerical simulations, such as the analysis of the transition of a multicellular clock of coupled repressilators to synchrony that identified a minimal cell density as a requirement for synchronization (Garcia-Ojalvo et al. 2004; Misra and Mitra 2008). Similar methods, combining theory and experimentation, were applied to populations of coupled chemical oscillators (Taylor et al. 2009). However, few analytic approaches to the synchronization problem exist. Model simplification and classical dynamic systems analysis of nullclines or bifurcations were used as alternative approaches to study synchronization in genetic relaxation oscillators (McMillen et al. 2002; 
Kuznetsov et al. 2004) and in simplified circadian clock models (Gonze et al. 2005; Locke et al. 2008), but these methods are limited to low-dimensional systems. Other previous works employed the Lur'e system approach to derive sufficient conditions for robust synchronization of coupled repressilators under stochastic perturbations (Li et al. 2007). Contraction theory was applied by Russo and di Bernardo (2009) to develop a method for the construction of synthetic biological clocks, and to apply it to a system of coupled repressilators (see also Wang and Slotine 2005). Lastly, multistability, clustering and noise-induced suppression of synthetic biological relaxation oscillators have been analyzed by Koseska et al. (2007a, 2007b).

Even though these studies provide valuable information about populations of synchronizing biomolecular oscillators, such as the effects of noise (e.g. Li et al. 2007; Zhou et al. 2008) or cell density (e.g. Zhou et al. 2008; Garcia-Ojalvo et al. 2004), a quantitative approach allowing for the comparison of synchronization performances of different network structures of synthetic oscillators is still missing. Thus, the design process has to rely on vast numbers of simulations that do not reveal underlying reasons for good or bad performance, and their interplay (e.g. Misra and Mitra 2008; Zhou et al. 2008). Furthermore, cell-to-cell variability leading to a distribution of eigenfrequencies of the oscillators is often solely addressed by the addition of white noise (e.g. Russo and di Bernardo 2009; Zhou et al. 2008), the cell environment is assumed to be well mixed (e.g. McMillen et al. 2002; Kuznetsov et al. 2004), and only requirements for full synchronization are derived (e.g. Li et al. 2007; Russo and di Bernardo 2009). In cases where partial synchronization is sufficient, these requirements might be too conservative.

In this article we develop a method that enables us (a) to analyze the properties of different possible network structures of synchronizing oscillators, (b) to determine the synchronization performance and, hence, (c) to systematically find the best network structure that may be implemented in vivo. Analysis of a generalized model enables us to deduce the major factors determining if a population of oscillators synchronizes, and to optimize the network structure and the parameter values one is able to modify. In addition, we analyze how spatial effects influence the synchronization. We explain the emergence of spatial phase waves whose suppression might be a crucial design requirement for naturally evolved as well as for engineered systems.

Our method is exemplified by analyzing and comparing three models of the mammalian oscillator recently developed by Tigges et al. (2009). The previously published model is extended differently by parts of the quorum sensing mechanism of Vibrio fisheri. We adapt this signaling mechanism to exchange phase-related information between the cells.

This article is organized as follows. In Sect. 2 we introduce a generalized normal form for chemically coupled synthetic oscillators and derive a formula quantifying the synchronization performance of a network in the normal form. We exemplify our results in Sect. 3 by calculating the synchronization performance of three competing network structures of coupled mammalian oscillators. In Sect. 4 we show that when the extracellular medium is not well mixed, spatial phase waves can occur, which may hinder synchronization significantly. Finally, in Sect. 5 we illustrate these phase waves with the help of spatial simulations of the population of coupled mammalian oscillators. 


\section{Synchronization Performance}

Most synchronizing networks of synthetic oscillators have structural similarities. Commonly the network in cell $i$, where $i=1, \ldots, N$, consists of a set of intracellular species $\mathbf{x}_{i}$ (e.g. mRNAs and proteins) that form the core oscillator. If the core oscillator was implemented alone, each cell would exhibit regular oscillations, but it would not synchronize to other cells. To allow the cells to synchronize, the network is extended by a signaling molecule $s_{i}$. This signaling molecule can diffuse through the cellular membrane and it is used to transfer information about the current phase of the oscillator in cell $i$ to surrounding cells. Inside the cell, $s_{i}$ can react with one or more of the intracellular species $\mathbf{x}_{i}$, driving the phase of the oscillator towards synchrony with the other cells.

In the following we propose a standard form to which many of the recently published models (McMillen et al. 2002; Garcia-Ojalvo et al. 2004; Kuznetsov et al. 2004; Li et al. 2007; Zhou et al. 2008; Russo and di Bernardo 2009) of autonomously synchronizing cellular oscillators can be generalized. We show that all models compatible with this standard form and fulfilling some additional requirements can be reduced to their respective phase description. The phase description was primarily developed by Winfree (1967) and Kuramoto et al. (Kuramoto 1984; Kuramoto and Nishikawa 1987; Strogatz 2000). It is a simplified representation of the model by only one state per oscillator, the phase $\phi$, which represents the current position of the oscillator on its limit cycle.

\subsection{Background: Phase Description}

Kuramoto (1984) introduced a general system of $N \gg 1$ mutually coupled oscillators that is mathematically described by

$$
\frac{d}{d t} \mathbf{X}_{i}=\mathbf{F}_{i}\left(\mathbf{X}_{i}\right)-\sum_{j=1}^{N} \mathbf{V}_{i, j}\left(\mathbf{X}_{i}, \mathbf{X}_{j}\right), \quad i=1, \ldots, N
$$

where $\mathbf{X}_{i}$ is the state vector of the $i$ th oscillator, $\mathbf{F}_{i}$ its autonomous dynamics, and $\mathbf{V}_{i, j}$ a function describing the coupling between the $i$ th and the $j$ th oscillator. Each oscillator is assumed to have similar dynamics

$$
\mathbf{F}_{i}\left(\mathbf{X}_{i}\right)=\mathbf{F}\left(\mathbf{X}_{i}\right)+\Delta \mathbf{F}_{i}\left(\mathbf{X}_{i}\right), \quad \text { with } \Delta \mathbf{F}_{i}\left(\mathbf{X}_{i}\right) \text { small, }
$$

and the coupling between the oscillators has to be weak (meaning $\mathbf{V}_{i, j}$ is small). Furthermore, it is required that a hypothetical uncoupled oscillator with nominal dynamics

$$
\frac{d}{d t} \mathbf{X}=\mathbf{F}(\mathbf{X})
$$

to which we shall refer as the nominal oscillator, has to have a stable and robust limit cycle $\mathcal{C}$ with an associated period length $T$. In this context robust means that the states of the real oscillators (1) stay in a small vicinity of this limit cycle. Every state $\mathbf{X}_{0} \in \mathcal{C}$ on the orbit $\mathcal{C}$ is associated with a distinct scalar variable, namely its phase $\phi$. 
The mapping of the phase $\phi \in[0, T)$ to its associated state $\mathbf{X} \in \mathcal{C}, \mathbf{X}_{0}:[0, T) \rightarrow \mathcal{C}$ is defined such that the phase $\phi_{0}$ of the nominal oscillator linearly increases with time:

$$
\frac{\partial \phi_{0}}{\partial t}=1
$$

The mapping $\mathbf{X}_{0}(\phi)$ can be obtained by simulating the nominal oscillator (3) for one period length $T$, starting at an arbitrary time $t_{0}$ after it has reached the limit cycle $\mathcal{C}$, and setting $\phi:=t-t_{0}$.

To also associate a phase with every state of the non-nominal oscillators (1), the phase reduction method of Kuramoto (1984) is based on perturbation theory, where $\Delta \mathbf{F}_{i}$ and $\mathbf{V}_{i, j}$ are interpreted as small perturbations to the nominal dynamics $\mathbf{F}$. The phase $\phi_{i}$ of an oscillator (1) can then be approximated by

$$
\frac{d}{d t} \phi_{i}=1+\mathbf{Z}\left(\phi_{i}\right)\left(\Delta \mathbf{F}_{i}\left(\mathbf{X}_{0}\left(\phi_{i}\right)\right)-\sum_{j=1}^{N} \mathbf{V}_{i, j}\left(\mathbf{X}_{0}\left(\phi_{i}\right), \mathbf{X}_{0}\left(\phi_{j}\right)\right)\right)
$$

with $\mathbf{Z}(\phi)=\left.\operatorname{grad}_{\mathbf{X}} \phi\right|_{\mathbf{X}=\mathbf{X}_{0}(\phi)}$ being the phase-dependent sensitivity.

Due to weak coupling, the effect of the small perturbations on the phase $\phi_{i}$ of the $i$ th oscillator can be averaged over one period $T$. This results in

$$
\frac{d}{d t} \phi_{i}=1+\Delta \omega_{i}-\sum_{j=1}^{N} \Gamma_{i, j}\left(\phi_{i}-\phi_{j}\right), \quad i=1, \ldots, N,
$$

with $\Gamma_{i, j}$ the coupling function and $\omega=1+\Delta \omega_{i}$ the eigenfrequency of the $i$ th oscillator:

$$
\begin{aligned}
\Gamma_{i, j}\left(\Delta \phi_{i, j}\right) & =\frac{1}{T} \int_{0}^{T} \mathbf{Z}\left(\tau+\Delta \phi_{i, j}\right) \mathbf{V}_{i, j}\left(\mathbf{X}_{0}\left(\tau+\Delta \phi_{i, j}\right), \mathbf{X}_{0}(\tau)\right) d \tau, \\
\Delta \omega_{i} & =\frac{1}{T} \int_{0}^{T} \mathbf{Z}(\tau) \Delta \mathbf{F}_{i}\left(\mathbf{X}_{0}(\tau)\right) d \tau .
\end{aligned}
$$

The values of $\Gamma_{i, j}\left(\Delta \phi_{i, j}\right), \Delta \phi_{i, j}=\phi_{i}-\phi_{j}$, may be interpreted as follows: if $\Gamma_{i, j}\left(\Delta \phi_{i, j}\right)>0$ the frequency of the $i$ th oscillator decreases due to its coupling to the $j$ th oscillator, and if $\Gamma_{i, j}\left(\Delta \phi_{i, j}\right)<0$ the frequency increases. One can expect an attractive coupling of the oscillators if $\Gamma_{i, j}$ increases around the origin, and a repulsive coupling if $\Gamma_{i, j}$ decreases. In general, the larger the absolute minimal and maximal values of $\Gamma_{i, j}$, the higher the chance that a population of coupled oscillators synchronizes, and the better the synchronization results. Moreover, to achieve good robustness properties, it is additionally favorable that $\Gamma_{i, j}$ should be approximately (point-) symmetric for phase differences larger than zero in absolute value.

Even though all systems of coupled oscillators of the form of (1) satisfying the conditions mentioned above can be reduced to their phase description (6), this description is too complex to be analyzed analytically. Kuramoto (1984) thus concentrated on a special case of (6), where the coupling function has the following form:

$$
\Gamma_{i, j}\left(\phi_{i}-\phi_{j}\right)=\frac{1}{N} \kappa \sin \left(\frac{\phi_{i}-\phi_{j}}{T}\right),
$$


with $\kappa \in \mathbb{R}_{+}$being the coupling strength. Furthermore, the distribution $\Pi(\omega)$ of the eigenfrequencies is assumed to be symmetric and known, with $\Pi_{\max }$ denoting its maximum.

The order parameter $\varpi$ is introduced as a measure for the synchronicity of a population of oscillators, defined as

$$
\varpi(t)=\frac{1}{N}\left|\sum_{i=1}^{N} \exp \left(i \phi_{i}(t)\right)\right|, \quad \text { with } i^{2}=-1 .
$$

For a uniformly synchronized population, the order parameter $\varpi$ is one, whereas for a completely desynchronized population, it is zero. By calculating the bifurcation point when the unsynchronized steady state of a population of oscillators $(\varpi=0)$ becomes instable, Kuramoto (1984) was able to conclude that the following equation has to be satisfied for a population of oscillators to synchronize:

$$
\frac{\pi}{2} \Pi_{\max } \kappa>1 .
$$

\subsection{Generalized Standard Form}

We analyze ordinary differential equation (ODE) models of chemically coupled synthetic oscillators in the following standard form:

$$
\begin{aligned}
\left(\begin{array}{c}
\frac{d}{d t} \mathbf{x}_{i} \\
\frac{d}{d t} s_{i}
\end{array}\right) & =\left(\begin{array}{c}
\mathbf{f}\left(\mathbf{x}_{i}, \theta_{i}\right)+\mathbf{g}_{1}\left(\mathbf{x}_{i}, s_{i}, \theta_{i}\right) \\
g_{2}\left(\mathbf{x}_{i}, s_{i}, \theta_{i}\right)-k_{D s i} s_{i}-\eta\left(s_{i}-s_{e}\right)
\end{array}\right), \quad i=1, \ldots, N, \\
\frac{d}{d t} s_{e} & =\frac{V_{\text {cell }}}{V_{\text {suspension }}} \sum_{i=1}^{N} \eta\left(s_{i}-s_{e}\right)-k_{D s e} s_{e},
\end{aligned}
$$

with $V_{\text {cell }}$ and $V_{\text {suspension }}$ the volume of a single cell and the total suspension volume, $s_{i}$ and $\mathbf{x}_{i}$ the concentrations of the signaling molecule and the other molecular species inside the $i$ th cell, $s_{e}$ the concentration of the signaling molecule in the extracellular space, $\eta$ the diffusion rate constant of the signaling molecule through the cell membrane, and $k_{D s i}$ and $k_{D s e}$ the degradation rate constants of the signaling molecule inside the cell and in the extracellular space, respectively. The dynamics of the species $\mathbf{x}_{i}$ and their interactions with the signaling molecule $s_{i}$ are described by the functions $\mathbf{f}, \mathbf{g}_{1}$ and $g_{2}$. We assume that each oscillator has a different set of parameters $\theta_{i}$, which are distributed around a nominal set of parameters $\theta_{0}$ with an associated set of probability density functions $\mathbf{p}(\theta ; \sigma)$, where $\sigma$ denotes parameters describing the shape of $\mathbf{p}$. First, we assume that the extracellular space is well mixed, so that the extracellular signaling molecule $s_{e}$ has no spatial dependencies.

The standard form (11) can be interpreted as follows: The dynamics $\mathbf{f}$ alone represent the dynamics of the core oscillator, when it is not coupled to other oscillators. Often a small network solely containing the dynamics $\mathbf{f}$ of the core oscillator is already implemented in vivo and the idea is to modify it in such a way that the oscillators can synchronize. This is done by extending the network to the form of (11) by adding a small signaling molecule to enable cell-cell communication. 
The dynamics $\mathbf{g}_{1}$ and $g_{2}$ then represent the state-dependent production rates of the signaling molecule $s_{i}$, and its reactions with the species $\mathbf{x}_{i}$ of the core oscillator, respectively. The signaling molecule can diffuse between the intracellular and extracellular space. The net flux of signaling molecules through the membrane of the $i$ th cell is thus proportional to the difference in the intra- and extracellular signaling molecule concentrations, as represented by the terms $\eta\left(s_{i}-s_{e}\right)$ in (11a) and (11b). The degradation rate constant $k_{D s e}$ in the extracellular space can be different from the intracellular one $\left(k_{D s i}\right)$. A non-zero flow velocity of the extracellular medium as well as active degradation of $s_{e}$ (e.g. due to quorum quenching, see Waters and Bassler 2005) make it possible to alter the value of $k_{D s e}$ and thus to potentially fine-tune synchronization performance in the experimental setup.

In the following, we will speak of $\mathcal{S}=\left(\mathbf{f}, \mathbf{g}_{1}, g_{2}\right)^{T}$ as the network structure, of $\rho=$ $N \frac{V_{\text {cell }}}{V_{\text {suspension }}}$ as the cell density, and of $\sigma=\left(\sigma_{1}, \sigma_{2}, \ldots\right)^{T}$ as the cell-to-cell variability.

As was mentioned above, the core oscillator is already constructed in vivo in many setups, and often there are logical choices for the signaling molecule $s_{i}$. The question is then: how can one choose the coupling terms $\mathbf{g}_{1}$ and $g_{2}$ to achieve best synchronization performance?

\subsection{Requirements}

To transform a model in the standard form (11) to its phase representation, and to analyze this representation with the methods developed by Kuramoto (1984) (see Sect. 2.1), several requirements have to be fulfilled. Many of these requirements are directly related to those of the method of phase description, but reformulated in terms of our standard form. We are only interested in determining whether a population of cells that is initially desynchronized will synchronize, as opposed to describing the entire synchronization process. Therefore, we can relax some of the requirements. This is especially important due to the high degree of nonlinearity inherent in most molecular oscillators, which might lead to significantly different oscillatory behaviors dependent on the degree of synchronization.

For a nearly completely desynchronized population of cells $(\varpi \ll 1)$, the external signaling molecule $s_{e}$ will only slightly oscillate around its steady-state value for a large enough amount of cells $(N \gg 1)$. The focus on the onset of synchronization allows us to essentially ignore that the shapes of the limit cycles $\mathcal{C}_{i}$ as well as the period lengths $T_{i}$ of the oscillators may vary when the order parameter increases. Thus, we can linearize the limit cycles around the steady-state value of $s_{e}$. As in Sect. 2.1, these limit cycles $\mathcal{C}_{i}$ have to be robust and similar to each other, a property which can many times only be validated numerically (see Supplementary Information). Furthermore, the coupling between the oscillators has to be weak during the onset of synchronization and the distribution of the eigenfrequencies of the oscillators should be unimodal and approximately symmetric.

To be able to transform our general form to its phase representation, it is required that at the onset of synchronization, namely when $s_{e}$ oscillates slightly, the power of the higher harmonics of $s_{e}$ should be small compared to the power of the first harmonic. Higher harmonics of the oscillations of the intracellular signaling molecule concentrations $s_{i}$ are likely to cancel each other out when mixing in the extracellular 
space after diffusing through the cell membrane (11b), as shown in the Supplementary Information.

\subsection{Transformation into the Phase Representation}

The first step of the transformation of the standard form (11) to its phase representation is based on the elimination of $s_{e}$ in the generalized model (11). For some networks it is appropriate to assume a quasi steady state (QSSA) in the dynamics of $s_{e}$. However, this assumption might break down for low cell densities $\rho$, or if the period length $T_{i}$ of the oscillators or the degradation rate constant $k_{D s e}$ of the extracellular signaling molecule $s_{e}$ are too small. In these cases, $s_{e}$ will oscillate with a lower amplitude in the exact solution than obtained by the QSSA, and it will have a nonzero phase delay $\varphi_{1} \neq 0$. The cell density-dependent phase delay can result in a network structure with optimal performance for certain intervals of the cell density $\rho$ only. To account for this effect, we take advantage of the linearity of (11b) and use an approach based on a frequency domain analysis (for the complete derivation refer to the Supplementary Information). With this approach the generalized model (11) can be approximated by

$$
\left(\begin{array}{c}
\frac{d}{d t} \mathbf{x}_{i} \\
\frac{d}{d t} s_{i}
\end{array}\right)=\left(\begin{array}{c}
\mathbf{f}\left(\mathbf{x}_{i}, \theta_{i}\right)+\mathbf{g}_{1}\left(\mathbf{x}_{i}, s_{i}, \theta_{i}\right) \\
g_{2}\left(\mathbf{x}_{i}, s_{i}, \theta_{i}\right)-\hat{k}_{D s i}\left(s_{i}\right)+\frac{A_{1}(\rho) \eta}{N} \sum_{j=1}^{N}\left(s_{j}\left(t-\Delta t_{1}(\rho)\right)-\bar{s}\right)
\end{array}\right),
$$

with

$$
\begin{aligned}
\hat{k}_{D s i}\left(s_{i}\right) & =\left(k_{D s i}+\eta\right) s_{i}-\eta A_{0}(\rho) \bar{s}, \\
A_{0}(\rho) & =\frac{\eta \rho}{k_{D s e}+\eta \rho}, \\
A_{1}(\rho) & =\frac{\eta \rho}{\sqrt{\frac{4 \pi^{2}}{T^{2}}+\left(k_{D s e}+\eta \rho\right)^{2}}}, \\
\varphi_{1}(\rho) & =\arctan \frac{2 \pi}{T\left(k_{D s e}+\eta \rho\right)}, \\
\Delta t_{1}(\rho) & =\frac{T}{2 \pi} \varphi_{1}(\rho)=\frac{T}{2 \pi} \arctan \frac{2 \pi}{T\left(k_{D s e}+\eta \rho\right)},
\end{aligned}
$$

with $\bar{s}$ the mean concentration of $s_{i}$. The value of $\hat{k}_{D s i}$ represents the effective degradation rate of $s_{i}$, caused by the physical degradation and by the net transport of $s_{i}$ through the cell membrane for constant $s_{e}$. An example of how the values of $A_{0}(\rho)$, $A_{1}(\rho)$ and $\Delta t_{1}(\rho)$ change for increasing or decreasing cell densities $\rho$ can be found in Fig. 1. It is worth noting that a QSSA would result in $A_{1}=A_{0}$ and $\Delta t_{1}, \varphi_{1}=0 \forall \rho$.

The nominal oscillator corresponding to (11) has nominal parameter values $\theta_{0}$ and it is part of an infinitely large population of completely desynchronized oscillators 


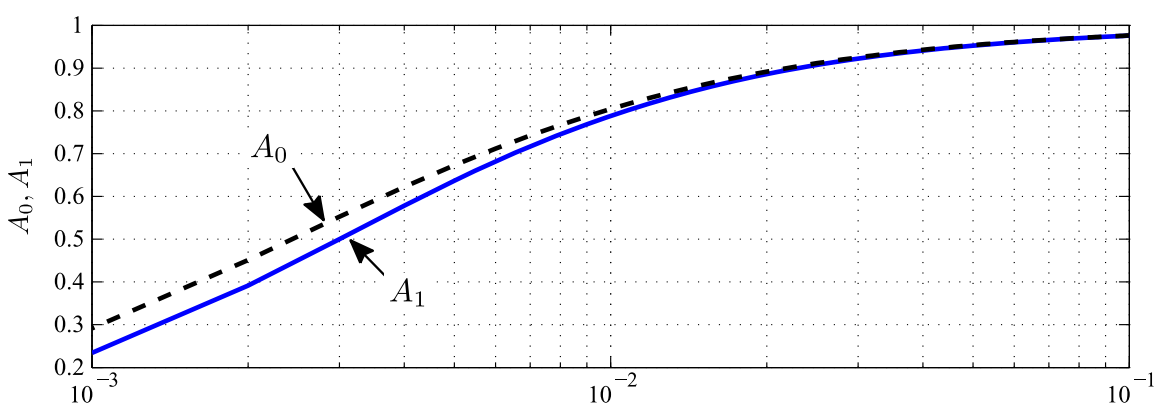

Cell density $\rho$

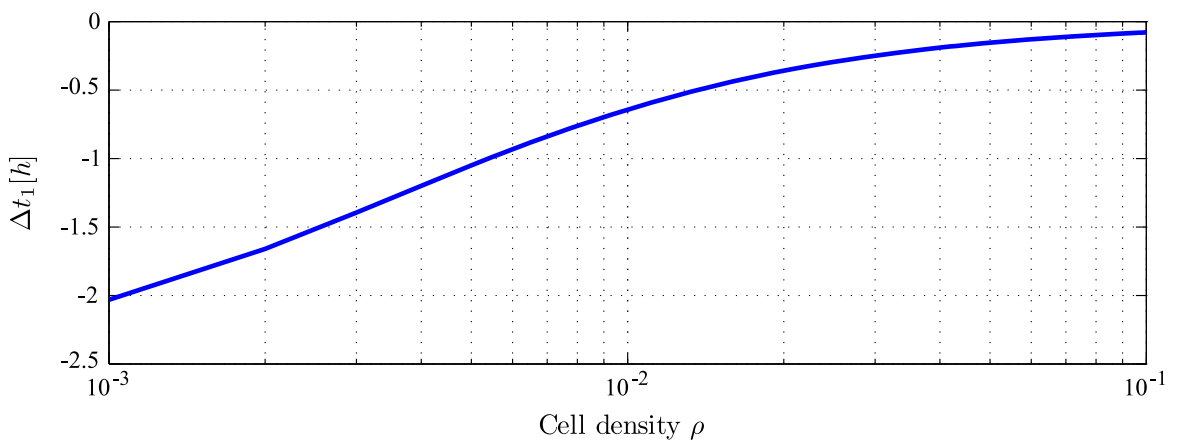

Fig. 1 Dependence of $A_{0}$ (black dashed, top), $A_{1}$ (blue solid, top) and $\Delta t_{1}$ (blue solid, bottom) on the cell density $\rho$ for oscillations with a period length of $T=20 \mathrm{~h}$, a degradation rate constant of the extracellular signaling molecule $s_{e}$ of $k_{D s e}=0.005 \mathrm{~min}^{-1}$, and a diffusion rate constant of $\eta=2 \mathrm{~min}^{-1}$ (see (13))

$(N \rightarrow \infty):$

$$
\underbrace{\left(\begin{array}{c}
\frac{d}{d t} \mathbf{x} \\
\frac{d}{d t} s
\end{array}\right)}_{=: \frac{d}{d t} \mathbf{X}}=\underbrace{\left(\begin{array}{c}
\mathbf{f}(\mathbf{x}, \theta)+\mathbf{g}_{1}(\mathbf{x}, s, \theta) \\
g_{2}(\mathbf{x}, s, \theta)-\hat{k}_{D s i}(s)
\end{array}\right)}_{=: \mathbf{F}(\mathbf{X})} .
$$

From the definition of $\hat{k}_{D s i}(13 \mathrm{a})$ it follows that not only the parameters $A_{1}$ and $\varphi_{1}$ (13) but also the dynamics of the nominal oscillator (14) might change with cell density. For certain network designs this can substantially change the dynamics of the nominal oscillator, which might eventually lead to the cancellation of-synchronized or unsynchronized-oscillations at certain cell densities. In general this effect is not desirable, but it can be reduced by a corresponding change in network design (see Sect. 3).

Following the approach used in Brown et al. (2004), the phase sensitivity $Z_{s}(t)$ is approximated numerically by using finite differences. It is obtained by perturbing $s$ (14) by a small value $\Delta s$ at time $t \in[0, T)$, and the phase difference, as compared to an unperturbed oscillator, is determined after the perturbed oscillator has returned to its orbit $\mathcal{C}$. The shape of $Z_{s}(t)$ can be approximated by solving the following implicit 
equation for a sufficiently high number of times $t_{j} \in[0, T)$ :

$$
Z_{s}\left(t_{j}\right) \mathbf{F}\left(\mathbf{X}_{0}\left(T_{\mathrm{end}}\right)\right)=\frac{\mathbf{X}_{\mathrm{pert}, t_{j}}\left(T_{\mathrm{end}}\right)-\mathbf{X}_{0}\left(T_{\mathrm{end}}\right)}{\Delta s},
$$

with $\mathbf{X}_{0}=\left(\mathbf{x}_{0}, s_{0}\right)^{T}$ the overall dynamics of the unperturbed oscillator, $\mathbf{X}_{\mathrm{pert}, t_{j}}=$ $\left(\mathbf{x}_{\text {pert }}, s_{\text {pert }}\right)^{T}$ the overall dynamics of the oscillator perturbed at time $t_{j}$, and $\Delta s$ the size of the perturbation.

We are now able to calculate the coupling function $\Gamma$ (see (7a)):

$$
\Gamma\left(\Delta \phi_{i, j}+\Delta t_{1}\right)=-\frac{A_{1} \eta}{N} \frac{1}{T} \int_{0}^{T} \mathbf{Z}_{s}\left(\tau+\Delta \phi_{i, j}+\Delta t_{1}\right)(s(\tau)-\bar{s}) d \tau .
$$

By approximating $\Gamma$ with a sine we obtain the two parameters representing the impact of the oscillator's structure $\mathcal{S}$ on the synchronization performance, the coupling strength $K_{1}(\mathcal{S})$ and the coupling timing $\lambda(\mathcal{S})$ :

$$
\Gamma\left(\Delta \phi_{i, j}+\Delta t_{1}\right) \approx \frac{A_{1} \eta}{N}\left(K_{0}+K_{1} \sin \left(\frac{2 \pi}{T} \Delta \phi_{i, j}+\lambda+\varphi_{1}\right)\right) .
$$

The value of $K_{0}$ should only deviate slightly from zero $\left(\left\|\eta A_{1} K_{0}\right\| \ll 1\right)$. If this is not the case it is likely that the value of $\bar{s}$ (see (12)) was not chosen correctly and should be adjusted.

Thus, our standard form (11) has the phase representation

$$
\frac{d}{d t} \phi_{i}=1+\Delta \omega_{i}-\frac{\eta A_{1}(\rho) K_{1}(\mathcal{S})}{N} \sum_{j=1}^{N} \sin \left(\frac{2 \pi}{T}\left(\phi_{i}-\phi_{j}\right)+\lambda(\mathcal{S})+\varphi_{1}(\rho)\right) .
$$

As in Sect. 2.1, the distribution $\Pi(\omega, \sigma)$ of the eigenfrequencies and its maximum value $\Pi_{\max }(\sigma)$ have to be known. In our setup they are fully determined by the probability density functions $\mathbf{p}(\theta, \sigma)$ of the distributions of the parameter values $\theta_{i}$. For most models it is impossible (or too time-consuming) to calculate the distribution $\Pi(\omega, \sigma)$ of the eigenfrequencies of the oscillators directly from the distributions of the parameter values $((2)$ and $(7 b))$. One then may use a Monte Carlo experiment and the relation

$$
\omega_{i}=1+\frac{T-T_{i}}{T_{i}}
$$

for approximating the shape and the maximum of $\Pi(\omega, \sigma)$. Alternatively, if the core oscillator is already implemented in vivo, $\Pi(\omega, \sigma)$ can be obtained by measuring the eigenfrequencies directly.

By using the results of Kuramoto (1984) on the requirements for the onset of synchronization of a network in its phase representation ((10), with $\left.\kappa=A_{1} \eta K_{1}\right)$ we propose a measure for the synchronization performance in the following section (for a more detailed derivation, please refer to the Supplementary Information). 


\subsection{Synchronization Performance}

For the generalized model of autonomously synchronizing oscillators (11) fulfilling the requirements described in Sect. 2.3 we can expect the onset of synchronization if the synchronization performance $\Sigma_{p}$ is greater than one:

$$
\Sigma_{p}:=\frac{\pi}{2} \Pi_{\max }(\sigma) \eta A_{1}(\rho) K_{1}(\mathcal{S}) \cos \left(\lambda(\mathcal{S})+\varphi_{1}(\rho)\right)>1 .
$$

The synchronization performance is a measure of the distance of a network from its bifurcation point, which lies at $\Sigma_{p}=1$. In general, the higher the value of the synchronization performance $\Sigma_{p}$, the better the expected synchronization outcomes and the higher the robustness (Kuramoto 1984). Thus, if one can choose between several possible network alternatives, the one with the highest synchronization performance $\Sigma_{p}$ is the most promising candidate to show good synchronization properties.

Three parameters - the cell density $\rho$, the network structure $\mathcal{S}$, and the cell-tocell variability $\sigma$-determine whether a population of chemically coupled cells (11) will synchronize. All of them have a multiplicative effect on the synchronization performance. Parameters $\rho$ and $\mathcal{S}$ have an additional joint relevance: the sum of $\lambda(\mathcal{S})$ and $\varphi_{1}(\rho)$ can be thought of as the coupling timing which determines if the oscillators are coupled attractively $\left(-\frac{\pi}{2}<\lambda+\varphi_{1}<\frac{\pi}{2}\right)$ or repulsively $\left(\left\|\lambda+\varphi_{1}\right\|>\frac{\pi}{2}\right)$. Since good synchronization performance can only be expected if the coupling timing is close to zero, the range of cell densities at which the synthetic oscillators operate should be defined prior to the search for the best network structure, as mentioned above.

The value of the synchronization performance might be biased for high values of $\left\|\lambda+\varphi_{1}\right\|$ (see Supplementary Information). However, since only networks with small coupling timings can be expected to show good synchronization results, network designs with high coupling timings are normally discarded.

The possibility to identify the influence of the cell-to-cell variability $\sigma$, the cell density $\rho$ and the network structure $\mathcal{S}$ on the synchronization performance and to predict if a given network of oscillators will synchronize are the main benefits of utilizing our method. We can thus identify the network structure with the best expected synchronization properties, but also systematically optimize a network structure for synchronization. For most networks, the cell-to-cell variability $\sigma$ is hard to influence, while the cell density $\rho$ is determined by cell culture properties and the intended application of the synthetic network. Thus, in the engineering process one should choose a network structure which maximizes $K_{1}$ and minimizes the absolute value of $\lambda+\varphi_{1}$.

\section{Example: Synchronization Performances of Three Different Networks of Mammalian Oscillators}

\subsection{Biological and Mathematical Setup}

We consider three different network structures $\left(\mathcal{S}_{1}, \mathcal{S}_{2}\right.$, and $\left.\mathcal{S}_{3}\right)$ of autonomously synchronizing synthetic mammalian oscillators. The networks consist of a core oscillator 


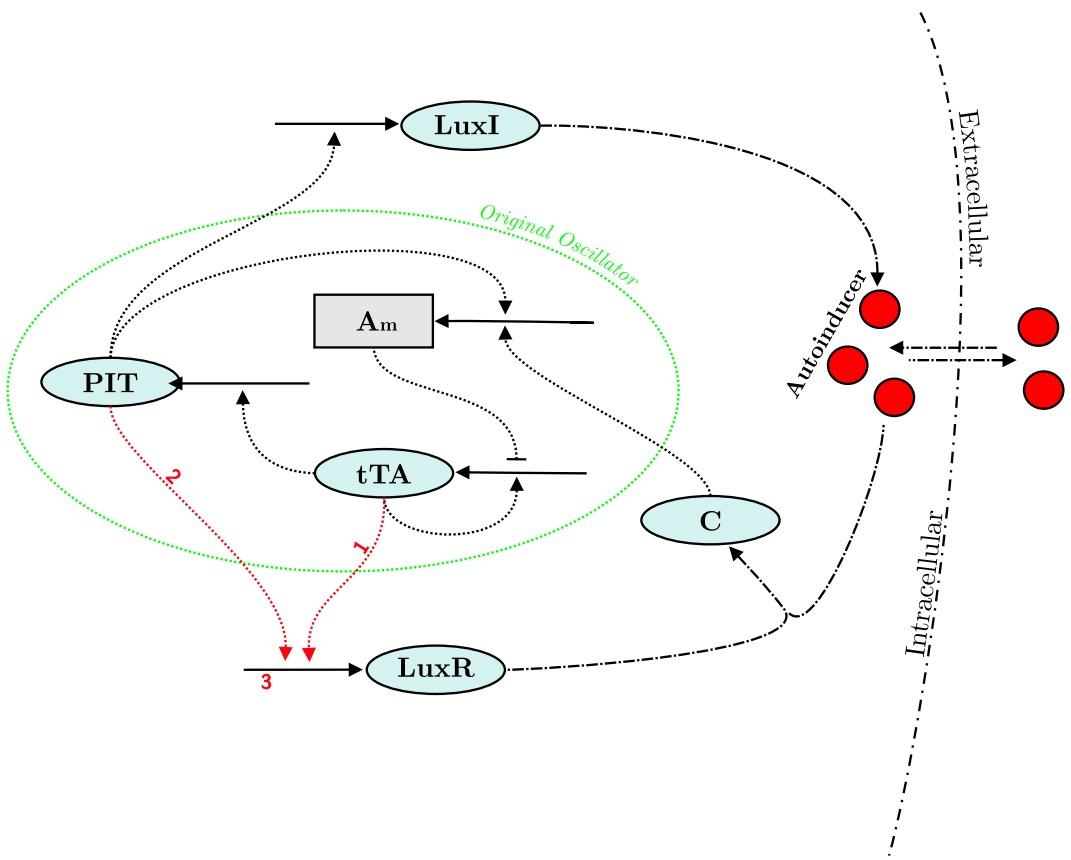

Fig. 2 Scheme of the structure of the oscillator models $\left(\mathcal{S}_{1}, \mathcal{S}_{2}\right.$ and $\left.\mathcal{S}_{3}\right)$ allowing autonomous synchronization of a population of oscillators through the autoinducer (red circle). The autoinducer is produced by LuxI, detected by LuxR, and can freely diffuse through the cell membrane. Solid black arrows: Transcription and translation. Dotted black and red arrows: Transcriptional activation. Dotted black "bar shaped" arrow: The antisense mRNA binds to the tTA mRNA. In the complex, the tTA mRNA is translationally inhibited. Dash-dotted black arrows: Production of the autoinducer, diffusion of the autoinducer through the cell membrane and dimerization of the autoinducer with the LuxR receptor protein. The transcriptional activation represented by the red arrows is only present in the respective models indicated by the numbers above the arrows

that is based on the existing synthetic oscillator of Tigges et al. (2009) in a combination with a communication module that incorporates parts of the quorum sensing mechanism of Vibrio fisheri (Goryachev et al. 2006).

The core oscillator (see Fig. 2) consists of two proteins, the tetracycline-dependent transactivator (tTA) and the pristinamycin-dependent transactivator (PIT), their respective mRNAs, and a tTA antisense mRNA. An antisense mRNA is a non-coding mRNA which has a complementary nucleotide sequence to its respective sense mRNA. The tTA antisense mRNA can bind to the tTA sense mRNA and thus deactivate its translation. Both the transcription of tTA mRNA and PIT mRNA is driven by the tTA protein, while the transcription of the tTA antisense mRNA is driven by the PIT protein. Furthermore, a reporter fluorescence protein is included in the network. Its gene has the same promoter as the tTA gene and is thus transcribed approximately at the same rate. Overall, thus, the core oscillator incorporates two feedback loops: a positive feedback via tTA and a time-delayed negative feedback through tTA and PIT. For more details on the core oscillator please refer to Tigges et al. (2009).

We extended the core oscillator to enable cell-to-cell communication through the production of an entraining extracellular signal. This is achieved by an additional 
feedback loop utilizing the quorum sensing mechanism of the marine bacterium Vibrio fisheri, consisting of two genes encoding the sender protein LuxI and the receptor protein LuxR. It has been shown that LuxR type proteins from bacteria can be engineered to also function as signal-dependent transcription factors in mammalian cells (Shiner et al. 2004). In all three models the transcription rate of the LuxI gene depends on the phase of the core oscillator through the PIT transcriptional activator. Since the LuxI protein synthesizes the autoinducer (3OC6HSL, a small signaling molecule), the concentration of the autoinducer will oscillate with the same frequency as the core oscillator, but with a phase shift depending on the dynamics of the transcription, translation and degradation of LuxI, and on the production and degradation rate of the autoinducer. Moreover, LuxR and the autoinducer form a complex that can be used as a transcriptional activator for additional genes.

A sensitivity analysis of the core oscillator revealed that its frequency is most sensitive to changes in the concentration of the antisense mRNA. This can be interpreted as follows: tTA activates its own transcription and it is bistable for certain concentrations of the antisense mRNA. When the antisense mRNA produced by the core oscillator is interpreted as the input and tTA as the output of the bistable subnetwork, its characteristic curve is a hysteresis. By adding antisense mRNA, the characteristic curve is shifted to the left and, by removing it, back to the right. By shifting the thresholds of the hysteresis at the right time, the oscillations can either be delayed or accelerated, which explains the high sensitivity of the frequency to perturbations in the antisense mRNA. Hence, in all three models we combined the gene of the antisense mRNA with a promoter activated by the receptor-autoinducer complex (see Fig. 2).

The difference in the three models arises from different activation mechanisms of LuxR transcription. In model $\mathcal{S}_{3}$ the LuxR gene is constitutively transcribed, whereas in models $\mathcal{S}_{1}$ and $\mathcal{S}_{2}$ the molecular species tTA and PIT are used as transcriptional activators, respectively. This results in either no oscillations in the concentrations of LuxR $\left(\mathcal{S}_{3}\right)$ or in oscillations with different phase shifts compared to the core oscillator $\left(\mathcal{S}_{1}\right.$ and $\left.\mathcal{S}_{2}\right)$.

It is known that variations in the plasmid copy numbers are the major source for differences in the parameter sets of oscillating cells (see Tigges et al. 2009). Thus, in all three models, every oscillator has distinct plasmid copy numbers, while we assume that all the other parameters have the same values in every cell. In order to minimize the effect of the uncertainty in the copy numbers, we combine the genes of the core oscillator on plasmid $P_{A}$ and the genes of the communication mechanism on plasmid $P_{B}$. The complete mathematical description of the dynamics of the models $\mathcal{S}_{1}-\mathcal{S}_{3}$ can be found in the Supplementary Information.

\subsection{Synchronization Performance of the Three Network Alternatives}

All three network alternatives (see Supplementary Information) are in the form of (11), with $s_{i}$ and $s_{e}$ the intra- and extra-cellular autoinducer concentrations, and $\mathbf{x}_{i}$ the state vector of cell $i$ containing all the other species. The requirements for the application of our method (see Sect. 2.3) are validated in the Supplementary Information. We can thus approximate the respective synchronization performances $\Sigma_{p, 1}$, $\Sigma_{p, 2}$ and $\Sigma_{p, 3}$ of $\mathcal{S}_{1}, \mathcal{S}_{2}$ and $\mathcal{S}_{3}$ by using (20). 


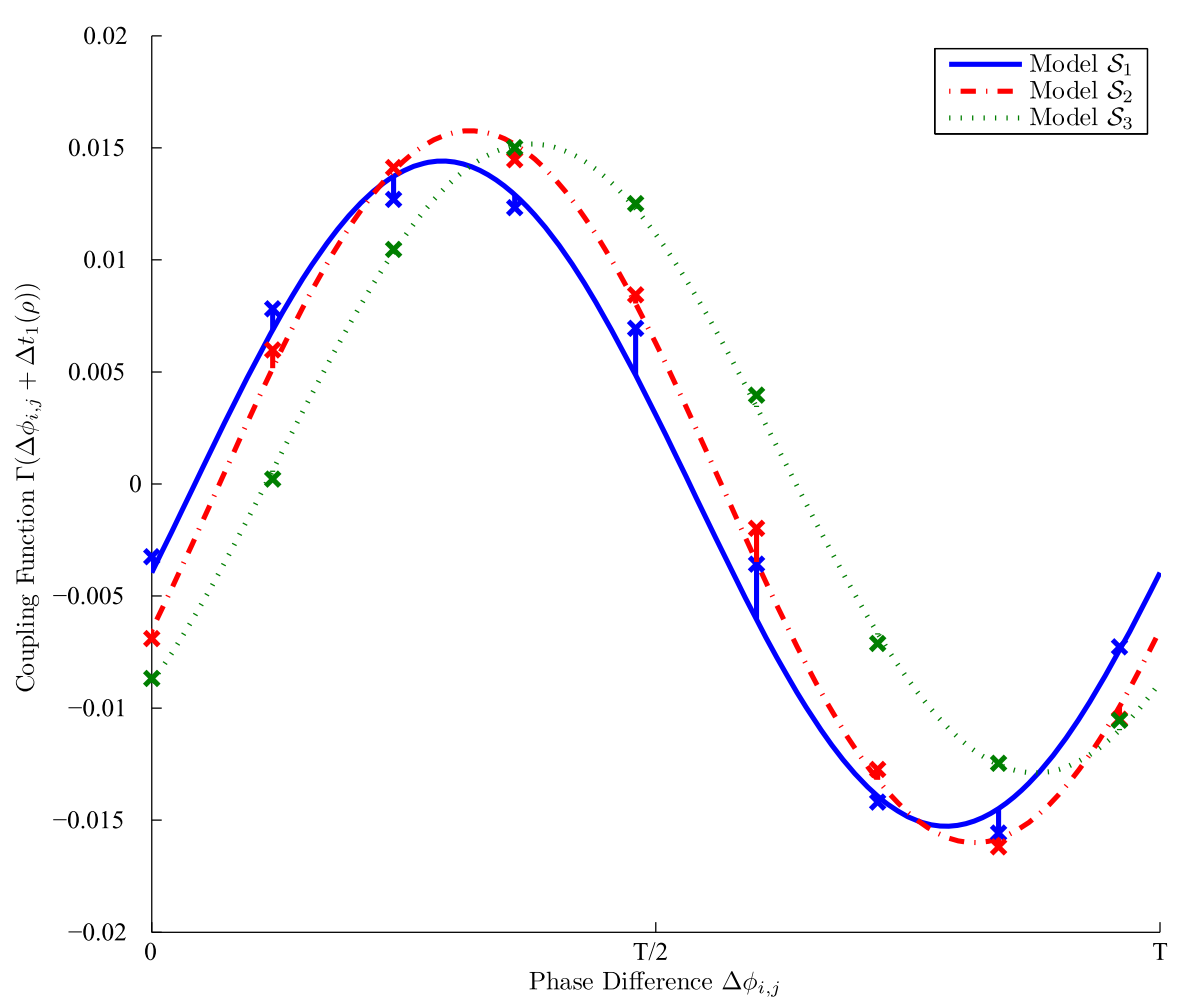

Fig. 3 The coupling functions $\Gamma\left(\Delta \phi_{i, j}+\varphi_{1}(\rho)\right)$ for the different models $\mathcal{S}_{1}$ (blue solid), $\mathcal{S}_{2}$ (red dash-dotted), and $\mathcal{S}_{3}$ (green dotted). Curves: Approximations of the synchronization forces by sines. Crosses: Data points calculated numerically, as described in Sect. 2.4

The dynamics of each oscillator are approximately $T$-periodic, with $T \approx 20 \mathrm{~h}$. In the following the cell density is set to $\rho=1 / 150$, which will fix the densitydependent variables (see Sect. 2.4) to $A_{0}=0.7278, A_{1}=0.6999$ and $\Delta t_{1}=0.669 \mathrm{~h}$.

Figure 3 shows the respective shapes of the coupling function $\Gamma$ (see (16)) obtained as described in Sect. 2.4. We approximate $\Gamma$ for the different models with sine functions (see (17)) to obtain the values for $K_{1}$ and $\lambda$ describing the influence of the network structure on the synchronization performance. For model $\mathcal{S}_{1}$ we obtain the values $K_{1}\left(\mathcal{S}_{1}\right)=0.015 \mathrm{~min}$ and $\lambda\left(\mathcal{S}_{1}\right)+\varphi_{1}(\rho)=-0.24$, for model $\mathcal{S}_{2}$ the values $K_{1}\left(\mathcal{S}_{2}\right)=0.016 \mathrm{~min}$ and $\lambda\left(\mathcal{S}_{2}\right)+\varphi_{1}(\rho)=-0.41$, and for model $\mathcal{S}_{3}$ the values $K_{1}\left(\mathcal{S}_{3}\right)=0.014 \mathrm{~min}$ and $\lambda\left(\mathcal{S}_{3}\right)+\varphi_{1}(\rho)=-0.79$. For each model the value of $K_{0}$ is negligible.

Note that the value of the coupling strength $K_{1}$ is very similar for all three models, whereas the coupling timing $\lambda+\varphi_{1}(\rho)$ of model $\mathcal{S}_{3}$ is substantially higher than the ones of $\mathcal{S}_{1}$ and $\mathcal{S}_{2}$, decreasing the synchronization performance of $\mathcal{S}_{3}$ significantly (see (20)).

Lastly, we obtained the distribution $\Pi_{\mathcal{S}_{1,2,3}}(\omega, \sigma)$ of the eigenfrequencies $\omega$ and their maximums $\Pi_{\max , \mathcal{S}_{1,2,3}}(\sigma)$ by Monte Carlo experiments with 5000 samples. The parameters with the largest fluctuations between different cells (and therefore the 


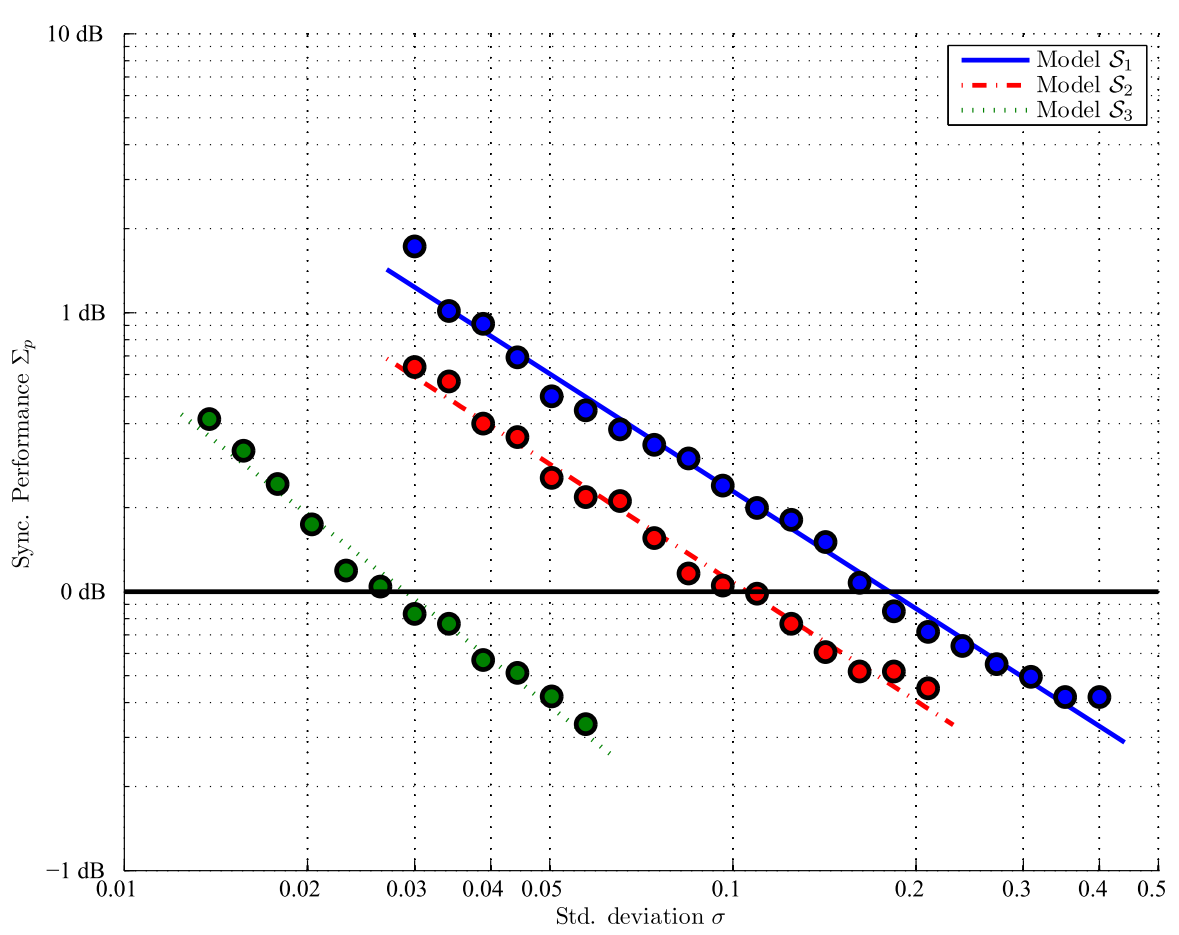

Fig. 4 Synchronization performances $\Sigma_{p, i}(\sigma), i=1, \ldots, 3$, of the models $\mathcal{S}_{1}$ (blue solid), $\mathcal{S}_{2}$ (red dash-dotted) and $\mathcal{S}_{3}$ (green dotted) for different values of the standard deviation $\sigma$ of the plasmid copy numbers. The curves are power-law fits to the data (markers). Above the black line one can expect the onset of synchronization as determined by (20)

determining factors for the distribution of the eigenfrequencies $\omega$ ) are the copy numbers of the plasmids $P_{A}$ and $P_{B}$ (Tigges et al. 2009). Because no sufficient models for plasmid infection exist, we assume the numbers of plasmids per cell to be uncorrelated and normally distributed around their nominal values with standard deviation $\sigma$. Experimental data for $\sigma$ is not available, and by consequence most of the results in this article are obtained for various standard deviations. For zero standard deviation $\Pi_{\max }(\sigma)$ is infinitely large and it monotonically decays for increasing values of standard deviation $\left(\frac{d}{d \sigma} \Pi_{\max }(\sigma) \leq 0\right)$.

We are now able to calculate the synchronization performance of all three models for different values of $\sigma$ by applying (20) (see Fig. 4). Our theory predicts that the network $\mathcal{S}_{1}$ will synchronize if the standard deviation $\sigma$ of the plasmid copy numbers is smaller than approximately $18 \%$. For network $\mathcal{S}_{2}$ we predict synchronization for $\sigma<11 \%$ and for $\mathcal{S}_{3}$ for $\sigma<3 \%$. However, note that for $\mathcal{S}_{3}$ the value of the coupling timing $\lambda+\varphi_{1}$ is relatively high. Thus, the value of the synchronization performance might be biased (see Sect. 2.5).

Network $\mathcal{S}_{1}$ thus shows the best synchronization performance, whereas network $\mathcal{S}_{3}$ is least suitable for autonomous synchronization. Besides the high value of $\lambda\left(\mathcal{S}_{3}\right)+\varphi_{1}(\rho)$, yet another effect decreases synchronization performance: In an unsynchronized population of cells where the extracellular autoinducer concentration 
shows only small deviations from the mean value, the eigenfrequencies of $\mathcal{S}_{3}$ have a higher dependency on the mean extracellular autoinducer concentrations, than those of models $\mathcal{S}_{1}$ and $\mathcal{S}_{2}$. For all three models cells stop oscillating if the extracellular autoinducer concentration is too high. However, for model $\mathcal{S}_{3}$ this point is reached already at a concentration of approximately $50 \mathrm{nM}$, in contrast to approximately $75 \mathrm{nM}$ for the other two models. The reason for this is the constitutive transcription of LuxR: if the mean autoinducer concentration has a high average value, a high amount of the antisense mRNA is transcribed constitutively in network $\mathcal{S}_{3}$ and the oscillators are likely to have a longer period, or even to stop oscillating altogether. On the other hand, even if the mean concentration of the autoinducer is relatively high in models $\mathcal{S}_{1}$ and $\mathcal{S}_{2}$, the oscillations are not interrupted, since the receptor protein LuxR is only transcribed if the oscillator is not blocked. An oscillating receptor protein concentration can thus increase robustness of the network, and lead to more homogeneous eigenfrequencies of the oscillators. We expect this result to be also applicable to other networks of synchronizing oscillators.

A larger absolute value of the coupling timing $\lambda(\mathcal{S})+\varphi_{1}(\rho)$ results in increased $\left(\lambda(\mathcal{S})+\varphi_{1}(\rho)<0\right)$ or decreased $\left(\lambda(\mathcal{S})+\varphi_{1}(\rho)>0\right)$ frequencies of the oscillators of the mean field at the onset of synchronization. Our simulations (data not shown) indeed suggest that the frequency of the mean field of model $\mathcal{S}_{3}$ increases significantly after synchronization, compared to the mean value of the eigenfrequencies of the oscillators. However, the frequency of the mean field of the other two models decreases during synchronization, suggesting that other effects not considered in our approximations might have some impact. Since our analysis concentrates on the onset of synchronization and it is not able to describe the complete synchronization process, a theoretical explanation for this effect is not provided.

\subsection{Simulation Results}

To validate the results obtained in the previous sections we ran several simulations of the models $\mathcal{S}_{1}, \mathcal{S}_{2}$ and $\mathcal{S}_{3}$. A plot showing the evolution of the reporter fluorescent protein concentration over time for model $\mathcal{S}_{1}$ can be found in the Supplementary Information.

To make the simulation data comparable to the theoretical results obtained in the previous section, we estimated the phase $\phi_{i}$ of an oscillator from the simulation data. We used the analytic signal concept (Rosenblum et al. 2001), assuming an approximately sinusoidal evolution of the concentration of the reporter protein $G_{a, i}$ :

$$
A(t) \exp \left(i \phi_{i}(t)\right)=G_{a, i}(t)+\mathcal{H}\left(G_{a, i}(t)\right),
$$

with $\mathcal{H}$ being the Hilbert transform, $G_{a, i}$ the concentration of the active fluorescent protein in the $i$ th cell, and $i^{2}=-1$ the imaginary unit.

Solving (21) for $\phi_{i}, i=1, \ldots, N$, yields the distribution of $\phi_{i}$ for different times. A typical example for the temporal evolution of the distribution of phases of model $\mathcal{S}_{1}$ initialized with a non-synchronized population of 1000 oscillators is shown in Fig. 5. After about 20 oscillation periods the cells can be considered synchronized.

Contrary to the theory (Kuramoto 1984), the order parameter $\varpi$ calculated numerically with the help of (21) and (9) does not reach a steady state in our simulations. 


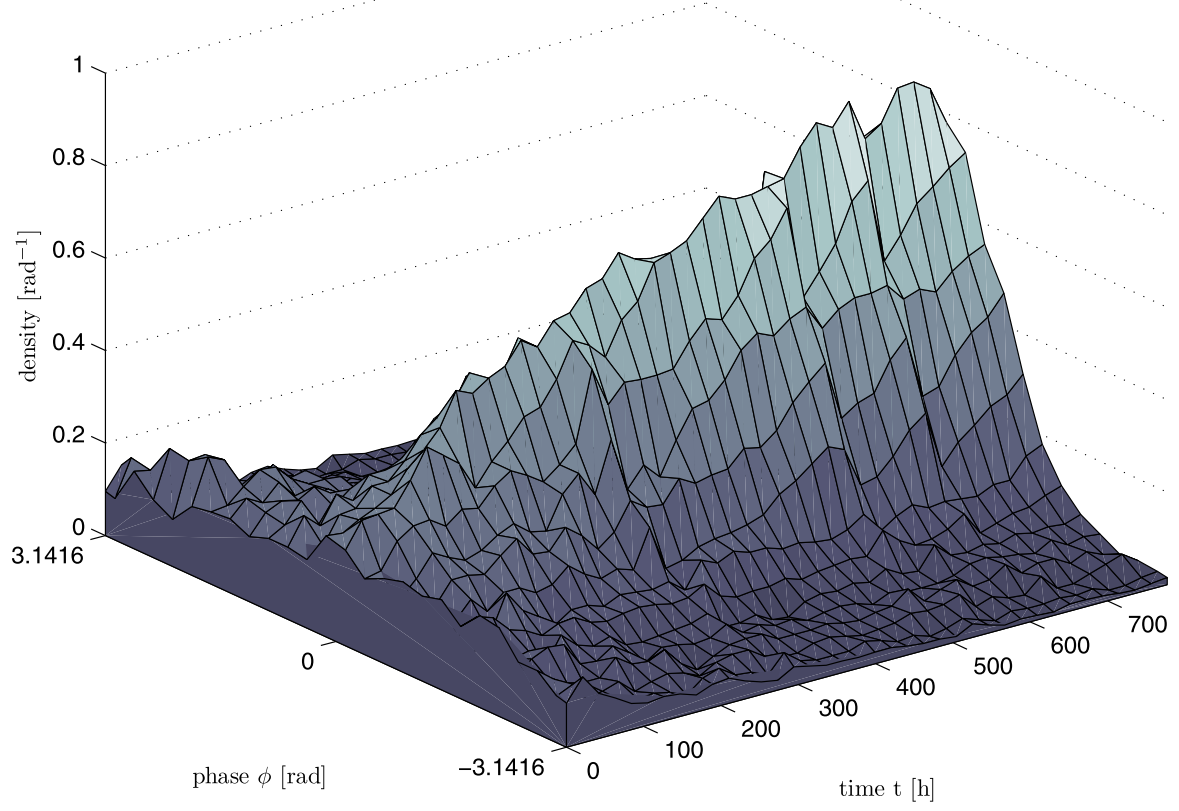

Fig. 5 Synchronization process of model $\mathcal{S}_{1}$. The simulation is based on a population of 1000 oscillators with Gaussian distributed plasmid copy numbers with a standard deviation of $15 \%$ of their nominal values. The plot shows the distribution of the phases $\phi_{i} \in(-\pi, \pi], i=1, \ldots, N$, of the oscillators at different times during the simulation. The oscillators were initialized to have approximately uniformly distributed phases and synchronize rapidly after the simulation started. For convenience, we shifted the distributions of the phases so that their maxima are at zero for every timestep

Instead, it shows regular small amplitude oscillations around some mean value with the same period length $T$ as the nominal oscillator (see Supplementary Information). These oscillations have several reasons: The "strength" with which a single oscillator is forced towards synchrony does not only depend on the difference in phase compared to the other oscillators, but also on the phase itself. This effect cannot be seen in the theory since the "strength" is averaged over one period (7). However, the major influence is probably the reconstruction of the phase of an oscillator (21) that is biased if the oscillations are not exactly sinusoidal. In the following we will thus take the mean value of $\varpi$ after the decay of the initial conditions as an approximation for its steady-state value.

We analyzed how the (averaged) steady-state value of the order parameter $\varpi$ changes for different standard deviations $\sigma$ of the plasmid copy numbers. For small standard deviations, the order parameter reaches a value close to one after the decay of the initial conditions (see Fig. 6). For high standard deviations, the order parameter decreases until it reaches an approximate steady value. The theory suggests that this steady value is zero (Kuramoto 1984). However, this is only true for an infinite number of oscillators. For a finite number of oscillators there are almost always a few oscillators that are synchronized by chance, and these 'perturbations' away from a 


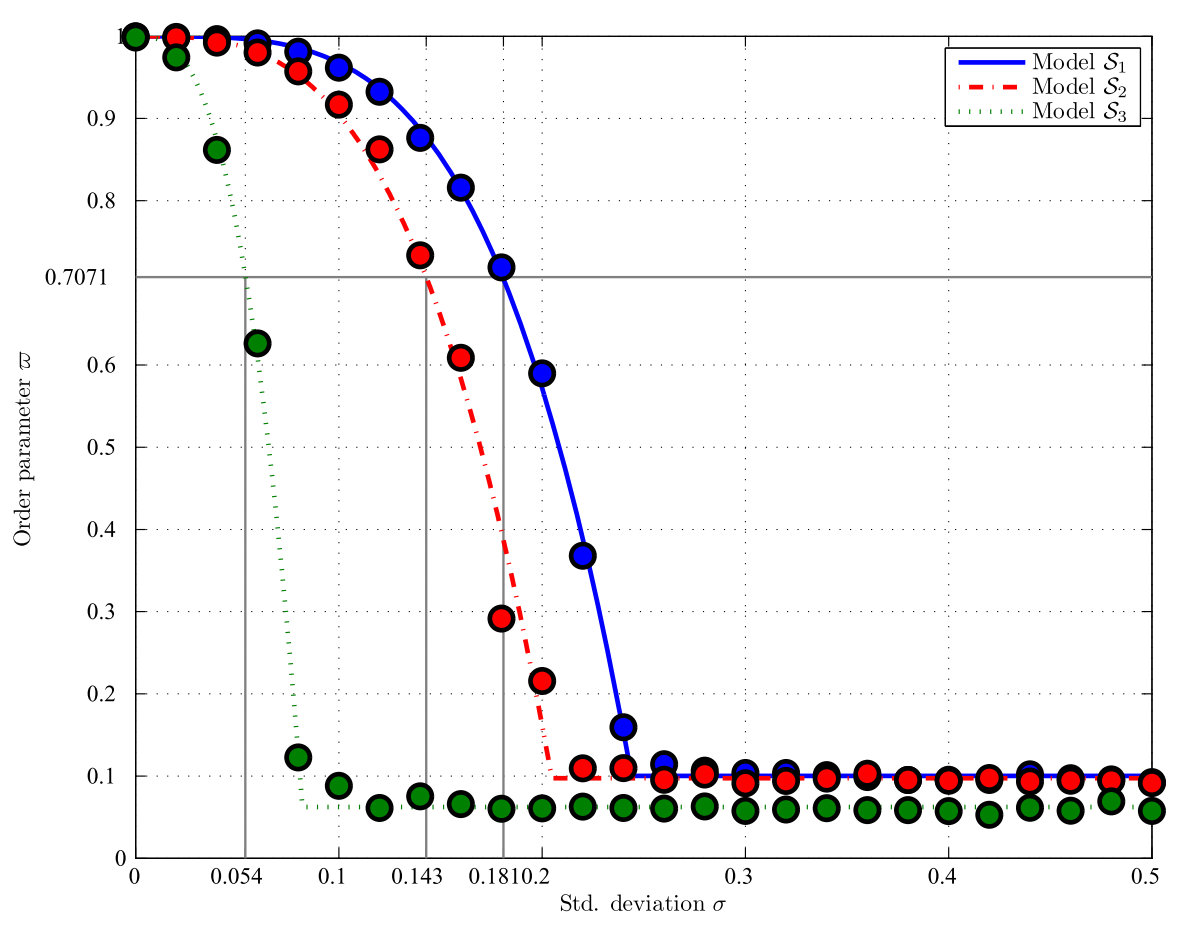

Fig. 6 The order parameter $\varpi(\sigma)$ as a function of the standard deviation $\sigma$ of the plasmid copy numbers for the three network designs. For each standard deviation $\sigma$ we run long-time simulations with a population of 2000 oscillators each. Since the order parameter is not constant after the decay of the initial conditions (see text), we display its mean value. Gray lines show the interpolations for order parameters of $\frac{1}{\sqrt{2}}$

completely non-synchronized state decay only slowly, so that the mean value of the order parameter never reaches zero.

We then approximated the evolution of the steady-state value of the order parameter by splines of the type $\varpi(\sigma) \approx \max \left(\sigma_{0}, 1-a \sigma^{k}\right)$, and calculated the critical standard deviations $\sigma_{C}$ where the order parameter decreases below $\frac{1}{\sqrt{2}}$. We obtained a $\sigma_{C}$ value of approximately 0.18 for model $\mathcal{S}_{1}, 0.14$ for model $\mathcal{S}_{2}$, and 0.05 for model $\mathcal{S}_{3}$. The values are in good agreement with the theoretical results obtained in Sect. 3.2, which predicted values of around 0.18 for $\mathcal{S}_{1}, 0.11$ for $\mathcal{S}_{2}$ and 0.03 for $\mathcal{S}_{3}$ (see Fig. 4). This illustrates that we can use the theory developed in the previous sections to distinguish between different network structures in order to find the structure with the best synchronization properties, as well as to predict the synchronization performance without the need for time-consuming simulations.

To explore the robustness of the network structure $\mathcal{S}_{1}$, as well as to further test the predictive power of the synchronization performance on the synchronization outcome, we performed a parameter space exploration (see Supplementary Information). We perturbed the parameters for which the model showed the highest sensitivity by varying them between 50 and $200 \%$ of their original value. For each perturbed parameter set we calculated the order parameter from simulations with 2000 cells as well 
as the synchronization performance with the help of (20). The model shows good robustness properties, and for parameter values associated with a high synchronization performance we observe a high order parameter after synchronization and vice versa.

The tendency of the order parameter to increase for standard deviations $\sigma$ slightly higher than the critical one predicted theoretically in Sect. 3.2 was already described before. In Daniels (2005), the authors analyzed the dependency of the onset of synchronization on the number of oscillators by several simulations with different population sizes. They observed that the onset of synchronization seems to be smoothed for small numbers of oscillators. In Sect. 3.2 we calculated $\sigma_{C}$ for the limit of an infinite number of oscillators, whereas in the simulations the number of oscillators is limited. One may interpret Fig. 6 as a numerical approximation of a bifurcation diagram, and the critical standard deviation $\sigma_{C}$ as an approximation of the bifurcation point. Only the stable solutions of the supercritical pitchfork bifurcation are depicted and, because the order parameter $\varpi$ has to be positive, the negative branch after the bifurcation disappears. Already near the bifurcation point the stability of the unsynchronized state decreases (the real part of at least one eigenvalue approaches zero). Hence, perturbations away from the unsynchronized state tend to be larger and to decrease more slowly, an effect that is generally more pronounced for lower numbers of oscillators. If the perturbations are large enough, the assumption that each oscillator is only slightly perturbed from its limit cycle due to the coupling (see Sect. 2.3) breaks down, which can result in a different value for the coupling strength $K_{1}$. Thus, due to a limited population size $N$, the bifurcation might appear to happen earlier. It may thus be reasonable to consider the critical standard deviation $\sigma_{C}$ as a lower bound for the value above which synchronization is not achieved.

\section{Spatial Phase Waves}

In Sects. 2 and 3 we assumed the signaling molecule $s_{e}$ in the extracellular space to be well mixed, which is often a viable assumption in small systems, or for bioreactors that are artificially mixed. Nevertheless, if this assumption does not hold, we have to refer to a spatial model with extracellular signaling molecule concentrations depending on the spatial position z. Such a spatial model takes diffusion and convection processes into account and it considers the production of the signaling molecule inside the single cells and its subsequent transport through the cell membrane.

In this section we show that phase waves can occur in a generalized spatial model, as was recently observed experimentally by Danino et al. (2010). Under certain conditions, such phase waves can significantly decrease the synchronization performance. Hence, they should be already considered in the design of synthetic multicellular oscillatory networks.

We assume that the cells are embedded in a two-dimensional biofilm, with the concentration of $s_{e}$ being nearly constant in the third dimension given the relative thinness of the biofilm. Moreover, we assume the cells to be substantially smaller than the two other dimensions, and the concentration of the signaling molecule $s_{e}$ to be nearly constant around each cell. As a simplification, we approximate the shape of the cells by spheres with radius $r_{\text {cell }}$. For many networks it is necessary to add an 
extracellular bulk flow either to "wash out" signaling molecules with a naturally high half-life, or to provide the cells with necessary nutrients.

As in Sect. 2, the dynamics inside each cell are described by a set of ODEs (see (11a)). However, in the extracellular space, we describe the signaling molecule concentration $s_{e}$ by following the approach in Müller et al. (2006) using a twodimensional advection-diffusion-reaction equation:

$$
\begin{aligned}
\frac{\partial}{\partial t} s_{e}(t, \mathbf{z})= & D \nabla^{2} s_{e}(t, \mathbf{z})-k_{D s e} s_{e}(t, \mathbf{z})-\vec{u}(\mathbf{z}) \cdot \nabla s_{e}(t, \mathbf{z}) \\
& +\frac{V_{\text {cell }}}{V_{\text {cylinder }}} \sum_{i=1}^{N} \delta\left(\mathbf{z}_{i}-\mathbf{z}\right) \eta\left(s_{i}(t)-s_{e}(t, \mathbf{z})\right)
\end{aligned}
$$

with $\mathbf{z}=\left(z_{1}, z_{2}\right)^{T} \in \mathbb{G}$ the spatial variable, $\mathbb{G} \subset \mathbb{R}^{2}, D$ the diffusion coefficient, $\vec{u}=$ $\left(u_{1}, u_{2}\right)^{T}$ the velocity of the bulk flow, $\mathbf{z}_{i}=\left(z_{i 1}, z_{i 2}\right)^{T} \in \mathbb{G}$ the position of the $i$ th cell, and $\nabla$ the Nabla operator. The constant $V_{\text {cylinder }}$ denotes the volume of a cylinder with the same radius $r_{\text {cell }}$ as the cells and the height of the biofilm. The function $\delta(\Delta \mathbf{z})$ is defined as follows:

$$
\delta(\Delta \mathbf{z})= \begin{cases}1 & \text { if }\|\Delta \mathbf{z}\|<r_{\text {cell }} \\ 0 & \text { otherwise }\end{cases}
$$

Similarly to Sect. 2.3 , we require that the states $\left(\mathbf{x}_{i}, s_{i}\right)^{T}$ of the oscillators move on a stable limit cycle $\mathcal{C}$ and that perturbations from this limit cycles due to spatial variations in $s_{e}$ are small.

In this section we assume that differences in the eigenfrequencies of the oscillators due to cell-to-cell variability, and due to different mean values of the extracellular signaling molecule play only a minor role for the occurrence of waves in a synchronized population of cells. Our simulations confirmed this assumption (see Sect. 5), except for the oscillators at the boundaries of the biofilm. Thus, only different eigenfrequencies of the oscillators at the boundaries are considered in our analysis.

To the best of our knowledge, there is no adequate analytic method to study a partial differential equation coupled to systems of ordinary differential equations, such as (22). Nevertheless, it is possible to get a deeper understanding of the dynamics of (22) by analyzing a simplified model. As a first simplification we assume to have many identical cells $(N \gg 1)$ equally distributed on a large rectangular plate, while each cell is assumed to be at an equal distance away from its neighboring cells. In what follows, we will analyze the long term behavior of the population only. Therefore the effect of initial conditions is only casually discussed, and we assume the cells to be locally synchronized so that oscillators near to each other have similar phases.

With these assumptions, we can coarse-grain (22) by changing the model to have a continuous cell density $\rho=$ const. This approximation is only feasible if the spatial and temporal dynamics are slow compared to the diffusion, such that the signaling molecule has a spatially and temporally differentiable concentration. Additionally, effects depending on the discrete nature of the cells should not significantly alter the coarse dynamics of the population. 
By introducing an extended state vector $\mathbf{X}=\left(\mathbf{x}, s_{i}, s_{e}\right)^{T}$ that incorporates the dynamics inside a cell as well as the dynamics of the signaling molecule in the extracellular space, we obtain the spatial model

$$
\begin{aligned}
\frac{\partial \mathbf{X}}{\partial t}=\left(\begin{array}{c}
\frac{\partial \mathbf{x}}{\partial t} \\
\frac{\partial s_{i}}{\partial t} \\
\frac{\partial s_{e}}{\partial t}
\end{array}\right)= & \underbrace{\left(\begin{array}{c}
\mathbf{f}(\mathbf{x})+\mathbf{g}_{1}\left(\mathbf{x}, s_{i}\right) \\
g_{2}\left(\mathbf{x}, s_{i}\right)-k_{D s i} s_{i} \\
-\left(k_{D s e}+k_{\mathrm{wash}}\right) s_{e}
\end{array}\right)+\left(\begin{array}{c}
\mathbf{0} \\
1 \\
-\rho
\end{array}\right) \eta\left(s_{e}-s_{i}\right)}_{=: \mathbf{F}(\mathbf{X})} \\
& +\left(\begin{array}{c}
\mathbf{0} \\
0 \\
1
\end{array}\right) \underbrace{\left(D \nabla^{2} s_{e}-\left(\vec{u} \cdot \nabla-k_{\mathrm{wash}}\right) s_{e}\right)}_{=: P\left(s_{e}\right)},
\end{aligned}
$$

with $k_{\text {wash }}$ the change in the apparent degradation constant of $s_{e}$ due to the "washing out" effect of the extracellular flow, defined as the mean value of $\vec{u} \cdot \nabla \ln s_{e}$. Note that the two terms including $k_{\text {wash }}$ sum up to zero; they are only introduced to achieve an approximately zero mean value of $P\left(s_{e}\right)$. Each element of the extended state vector $\mathbf{X}$ depends on the spatial position $\mathbf{z}$ even though it is not explicitly stated.

In almost the same manner as explained in Sect. 2.1, the term $P\left(s_{e}\right)$ can be seen as a small perturbation from the nominal dynamics $\mathbf{F}$ of the model, and the system can be transformed to its respective phase representation. By replacing the term containing $\Delta \mathbf{F}_{i}$ and $\mathbf{V}_{i, j}$ in (5) by $P\left(s_{e}\right)$ and subsequent averaging, we obtain a twodimensional partial differential equation (PDE) for the phase (see Kuramoto 1984):

$$
\frac{\partial \phi}{\partial t}=1+\alpha D \nabla^{2} \phi+\beta D(\nabla \phi)^{2}-\alpha \vec{u}(\mathbf{z}) \cdot \nabla \phi+\bar{\gamma},
$$

with the spatial coupling quantified by the parameters

$$
\begin{aligned}
& \alpha=\int_{0}^{T} Z_{s e}(\phi) \frac{\partial s_{e}}{\partial \phi} d \phi, \\
& \beta=\int_{0}^{T} Z_{s e}(\phi) \frac{\partial^{2} s_{e}}{\partial \phi^{2}} d \phi, \\
& \bar{\gamma}=k_{\text {wash }} \int_{0}^{T} Z_{s e}(\phi) s_{e}(\phi) d \phi,
\end{aligned}
$$

with the phase sensitivity $Z_{s e}(\phi)=\left.\operatorname{grad}_{s_{e}} \phi\right|_{\mathbf{X}=\mathbf{X}_{0}(\phi)}$ to perturbations in the extracellular signaling molecule concentration $s_{e}$.

Note that, as in Sect. 2.1, the perturbations of the oscillators away from $\mathcal{C}$ have to be small. Thus the value of the right-hand side of (25) has to be close to one. This requirement can be fulfilled either by a homogeneous spatial shape of the phase, by values of $\alpha, \beta$, and $\bar{\gamma}$ that are not too large (which implies weak coupling), or by a combination of both.

In what follows, $(25)$ is analyzed for a non-zero flow velocity without spatial dependence $(\vec{u}=$ const $)$. The flow enters the plate at its southern boundary and has zero 
velocity in the $z_{1}$-direction: $\vec{u}=\left(0, u_{2}\right)^{T}$. At the southern boundary the incoming flow has a relatively low mean signaling molecule concentration. In most networks this will either increase or decrease the eigenfrequencies of the oscillators near the boundary. We assumed the flow velocity $\|\vec{u}\|$ to be high enough, such that the signaling molecule in the extracellular space is mainly transported by advection. Thus the frequency of each cell is largely influenced by those of the cells to the south of it, rather than by cells in any other direction. Because no cells lie to the south of the boundary cells, it is unlikely that these cells will synchronize to a frequency significantly different from their own eigenfrequencies. We can thus expect the angular frequency at the southern boundary to be nearly constant $\left(\omega_{\text {inflow }}=\right.$ const $)$, which is confirmed by our simulation results (see Sect. 5). This can be mathematically described by a Dirichlet condition for the southern boundary:

$$
\varphi_{\text {south }}(\mathbf{z}, t, \phi, \vec{u})=\phi(\mathbf{z})-\omega_{\text {inflow }}(\vec{u}) t-\hat{\varphi}_{\text {south }}\left(z_{1}, \vec{u}\right)=0 .
$$

However, the spatial dependency $\hat{\varphi}_{\text {south }}\left(z_{1}, \vec{u}\right)$ in (27) cannot be determined without additional information.

For most networks it is not possible to pose adequate conditions for the three other boundaries. However, since the signaling molecule is mainly transported by advection, we expect that the other boundary conditions have only a weak effect on the oscillations of the cells far away from those boundaries.

In the Supplementary Information we show that, for a sufficiently large plate, the solution of (25) can be approximated for cells away from any border by

$$
\phi(t, \mathbf{z}) \approx \vec{\kappa}_{i} \mathbf{z}+\omega_{\text {inflow }}(\vec{u}) t+\phi_{0, i},
$$

with $\vec{\kappa}_{i} \in \mathbb{R}^{2}$ the wave vector, $\omega_{\text {inflow }}$ the angular frequency at the inflow, and $\phi_{0, i}$ the phase at $t=0$ which is determined by the initial conditions. The wave vector $\vec{\kappa}_{i}$ has to satisfy the following condition:

$$
\omega_{\text {inflow }}(\vec{u})=-\alpha \vec{\kappa}_{i} \cdot \vec{u}+\beta D\left\|\vec{\kappa}_{i}\right\|^{2}+(1+\bar{\gamma}) .
$$

If the eigenfrequency of the oscillators at the inflow is different from the mean eigenfrequency of the oscillators, due to different mean extracellular signaling molecule concentrations, it follows that $\vec{\kappa}_{i} \neq \overrightarrow{0}$. We then obtain a phase $\phi$ linearly increasing/decreasing with the spatial position $\mathbf{z}$, and linearly increasing with the time $t$. Such phases correspond to waves of the concentrations of the oscillators' species, to which we will refer as phase waves.

Both, phase waves in and contrary to the direction of the flow, are possible (29). However, in our simulations we only observed waves in the direction of the flow $\left(\vec{\kappa}_{i} \cdot \vec{u}<0\right)$. In this case, the size of the wave vector is approximately proportional to $\left\|1-\omega_{\text {inflow }}\right\|$ and inversely proportional to $\|\vec{u}\|$. To reduce the negative effect of waves in a small experimental setup, it might thus be favorable to chose a network structure that reduces the dependency of the eigenfrequencies of the oscillators on the mean extracellular autoinducer concentration (cf. Sect. 3.2), and to include an extracellular flow, even if it is not necessary to "wash out" the extracellular signaling molecule. 
It is biologically interesting to interpret the solution to the partial differential equation: From (28) we conclude that the cells at the southern inflow boundary act as pacemakers for the whole population of oscillators. Our simulations (see Sect. 5) suggest that once the pacemaker cells have synchronized, one can expect all cells to rapidly synchronize. However, if the southern boundary is large, it is possible that several local clusters of pacemaker cells evolve, containing cells synchronized to each other but not to cells of other clusters. Due to the assumptions made in our derivation, this effect cannot be described by the coarse-grained model (24). However, we anticipate a large time span until two or more clusters synchronize, since their communication is solely based on the diffusion normal to the flow.

In order to quickly obtain homogeneous oscillations, it is desirable to minimize the number of pacemaker cells to decrease the possibility of different evolving clusters. A broadening flow with spatially inhomogeneous velocity $\vec{u}=\vec{u}(\mathbf{z})$ leads to fewer pacemaker cells. Hence, the cells at the center of the southern boundary influence the majority of the other cells, while the cells at the edges of the southern boundary can only influence a few cells. Thus, we expect better synchronization properties of the whole population for a broadening flow.

In general it is not possible to find analytic closed solutions of (25) for spatial inhomogeneous flow velocities $\vec{u}(\mathbf{z})$. Even though we do not provide an analytical proof, we expect that the wavelength in this case has a dependence on the flow velocity $\vec{u}(\mathbf{z})$ similar to waves in media with a spatially homogeneous flow. Hence, if the flow is broadening and the flow velocity is high enough, we expect rapid synchronization and phase waves to evolve only in the direction of the flow, with a wavelength that can be larger than the dimensions of the plate. Thus, an apparently homogeneously synchronized population of spatially distributed oscillators can be achieved.

\section{Example: Phase Waves in a Population of Synchronizing Mammalian Oscillators}

In this section we will propose an experimental setup to achieve synchronization of a spatially distributed population of mammalian oscillator cells (see Sect. 3), and we will use numerical simulations to predict the synchronization process. The dynamics of the intracellular species $\mathbf{x}_{i}$ and $s_{i}$ are modeled according to Sect. 3.1 and the dynamics of the autoinducer follow an advection-diffusion-reaction equation (cf. (22)). The cells reside on a rectangular plate (see Fig. 7) where a flow of extracellular medium without autoinducers is entering with a constant flow velocity at the center of the left boundary; it leaves the plate and flows into a basin at the top and the bottom of the right boundary. The rest of the boundary is modeled as an impermeable wall, and the cells are assumed to be uniformly distributed on a small rectangular area placed in the middle-right side of the plate. To calculate the spatial and temporal diffusion process, we discretized (22) on a twodimensional ( $\left.n_{\text {grid }} \times m_{\text {grid }}\right)$ rectangular grid (Sewell 2005). The spatial derivatives are approximated by central differences and the time derivative is approximated by utilizing an explicit forward Euler scheme. The boundary conditions are set 


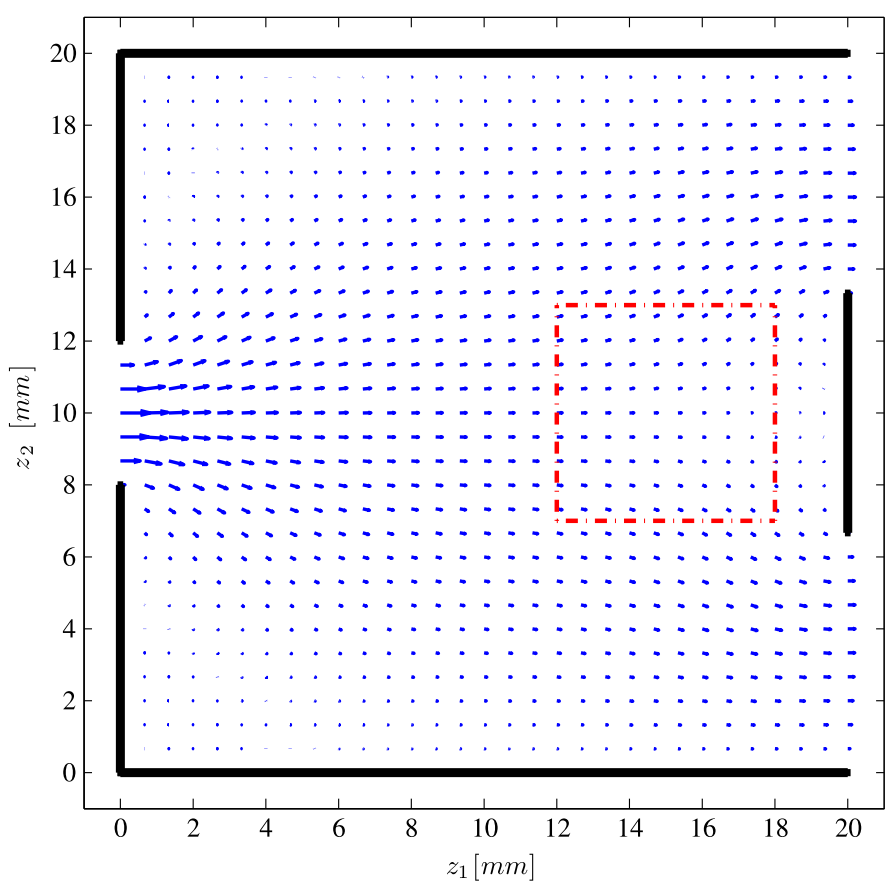

Fig. 7 We propose as an experimental setup a rectangular plate where the cells are uniformly distributed in the area surrounded by the red dotted line. The plate is assumed to be outlined by an impermeable wall (black solid lines). A broadening flow is entering the plate at an opening on the left side and leaving it through two openings on the right side

as follows: At the walls, the flux normal to the boundary is zero. The autoinducer concentrations at locations where the flow enters or leaves the plate are assumed to be zero. We obtained the flow velocity $\mathbf{u}_{j i}=\left(u_{1, j i}, u_{2, j i}\right)^{T}$ of the extracellular medium by solving the Navier-Stokes equations for incompressible viscous two-dimensional flow (see e.g. Acheson 1990) with the Semi-Implicit Method for Pressure-Linked Equations (SIMPLE, see e.g. Patankar and Spalding 1971; Ferziger and Peric 1999). For more details on the simulations we refer to the Supplementary Information. The resulting steady-state flow is shown in Fig. 7, where it should be noticed that the experimental setup leads to a broadening flow in the area where the cells are distributed, as suggested in Sect. 4.

Each cell has different plasmid copy numbers, with a standard deviation $\sigma$ of approximately $15 \%$ of the respective nominal copy number. Furthermore, the initial conditions were chosen such that the phases of the cells were approximately uniformly distributed. From our simulations it can be observed that the cells are basically synchronized after approximately 13 oscillation periods, when the majority of the cells oscillate with the same frequency and similar phases (see Fig. 8). A typical plot of the spatially distributed AHL concentrations in the extracellular space can be found in Fig. 9, where the onset of waves in the $z_{1}$-direction can be readily observed (see also Supplementary Videos). However, the wavelength is larger than the part of the plate where the cells are placed, so that the cooperative output signal of the pop- 


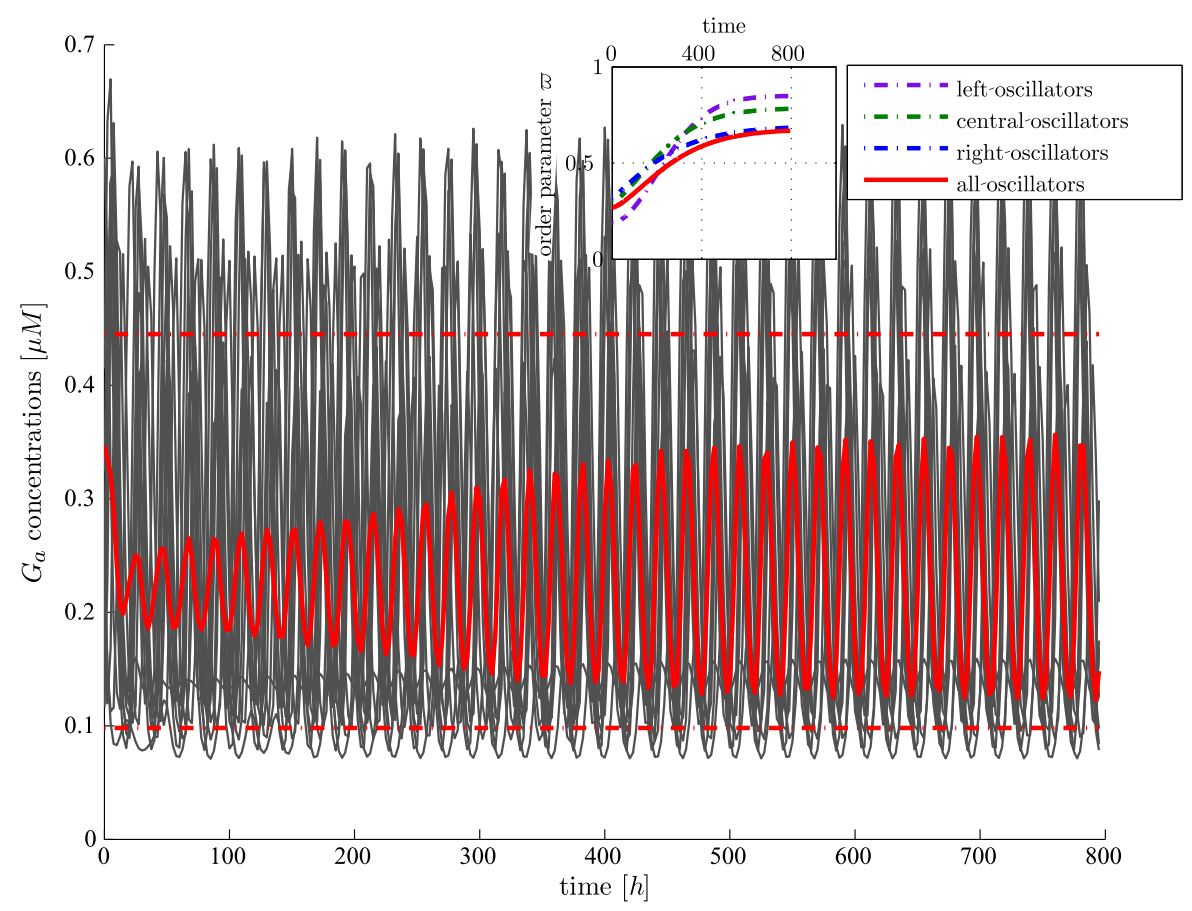

Fig. 8 Synchronization process of a population of 20,000 spatially distributed cells, with the plasmid copy numbers normally distributed with a standard deviation of $15 \%$ of their nominal values. Large image: The dark gray lines are the $G_{a}$ concentrations of 10 representatively chosen oscillators, the red curve is the mean $G_{a}$ concentration of all oscillators and the red-dotted lines are its maximal and minimal bounds, obtained by assuming perfect phase synchronization between the oscillators. Small image: Temporal evolution of the order parameter obtained as described in Sect. 3.3. The order parameter of the whole population (red curve) is most of the time lower than the local order parameters calculated only for the leftmost (purple dotted), central (green dotted) or the rightmost (blue dotted) third of the oscillators, which can be explained by the dependence of the phase on the spatial position due to the finite wavelength of the phase waves

ulation is clearly oscillating (green line in Fig. 8) as was anticipated in the previous section.

When plasmid copy numbers were chosen such that oscillators residing in the left third of the plate had a different eigenfrequency from that of all other cells, the frequency of the synchronized bulk oscillations was always close to the one of the cells facing the flow first, regardless of whether these cells had higher or lower eigenfrequencies than the rest of the cells. This effect is in agreement with the theoretical considerations in Sect. 4, which suggested that the cells first facing the flow act as pacemaker cells and force the other cells to a common frequency, thus enabling fast synchronization. On the other hand, the rest of the population seems to barely influence the frequency of the oscillations; it mainly increases the amplitude - the strength - of the signal. This observation might have interesting consequences for naturally evolved pacemakers: (a) identical cells may have different roles in synchronization depending on their spatial position, (b) oscillations do not necessarily become more regular when the amount of cells (but not their density) increases, and 

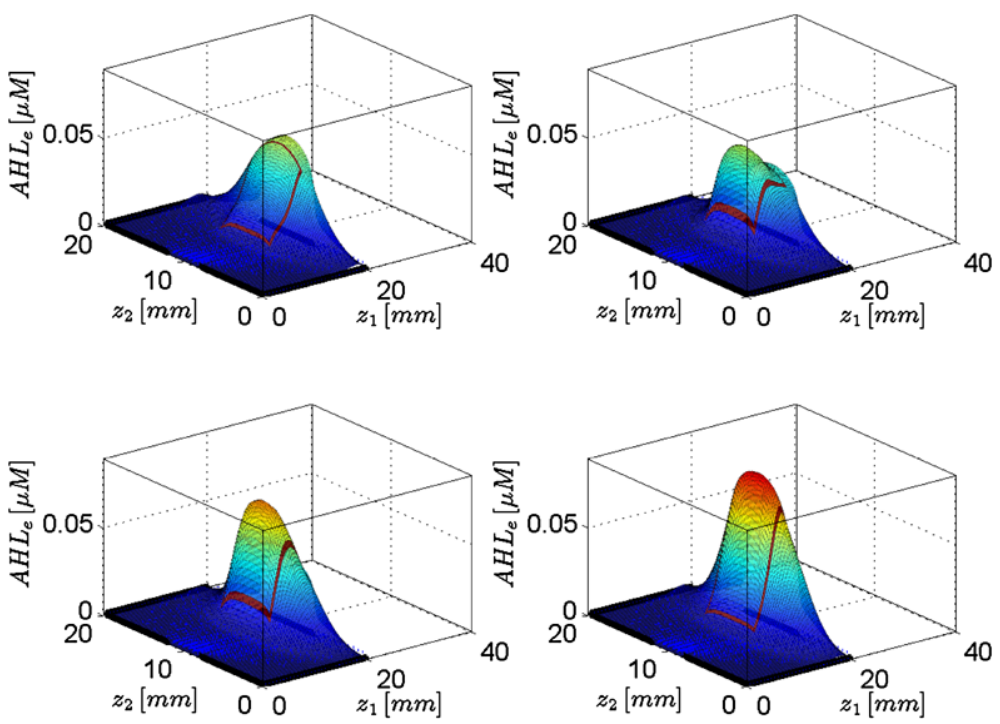

Fig. 9 Four images within one oscillation period of the extracellular concentration of AHL after synchronization. The onset of phase waves is already noticeable, although the wavelength is longer than the area of the plate where the cells are distributed (red rectangle)

(c) some cells inside a pacemaker might effectively be the "pacemakers of the pacemaker." These are interesting hypotheses, the study of which lies outside of the scope of this article.

\section{Summary and Conclusions}

We have developed a method to prescribe synchronization requirements for a population of synthetic single-cell oscillators, as well as to estimate their synchronization performance. The synchronization performance allows the comparison of competing network structures, and it supports the identification of a setup with the best synchronization properties for later implementation in vivo. We also introduced a normal form to which many synchronizing oscillatory networks can be generalized. It consists of a set of ordinary differential equations (ODEs) for every oscillator, and an ODE for an extracellular signaling molecule connecting the phases of the oscillators. For every oscillator we apply different parameter sets, thus allowing for a distribution of the oscillators' eigenfrequencies. To reduce the complexity of a model of several hundreds or thousands of such oscillators, we then applied the method of phase description, originally proposed by Kuramoto (1984) and Winfree (1967). This enabled us to derive requirements for the onset of synchronization as well as to decouple the impacts of cell density, of diversity of the oscillators, and of the applied coupling mechanism on the synchronization result.

More specifically, one of the major challenges for synchronizing synthetic oscillators in living cells is the vast cell-to-cell variability, resulting in a broad distribution of 
parameter values of the single oscillators. We have exemplified our method by considering an extended network based on the synthetic mammalian oscillator of Tigges et al. (2009). In this case, the determining factors for cell-to-cell variability are different plasmid copy numbers in each cell that lead to different eigenfrequencies of the oscillators. This cell-to-cell variability cannot be adequately modeled as white noise, and it has to be considered explicitly. This requirement has several implications. First, perfect phase synchronization is not feasible. Upon frequency synchronization, oscillators with large autonomous period lengths have phases lagging behind the mean phase, while oscillators with small autonomous periods have phases ahead of the mean phase of the population of oscillators. Also, if the parameter sets are widely distributed, there will be oscillators that do not synchronize their frequencies, such that only partial synchronization can be achieved.

The possibility to optimize a system of synthetic oscillators for autonomous synchronization makes our methodology a valuable design tool for synthetic biology. However, one has to iteratively shape the influence of the phase difference between two oscillators on their frequencies in order to achieve a better synchronization. This can be done by utilizing standard optimization algorithms, or manually if the number of design decisions is not excessively large. For the synthetic mammalian oscillator of Tigges et al. (2009) we conclude it to be advantageous to not only have a phasedependent production rate of the signaling molecule for communication, but also to engineer a phase-dependent production of the corresponding receptor protein. This might also hold true for naturally evolved oscillatory networks (Preitner et al. 2002).

It is important to note that our method is based on a model reduction, which might bias the results. We only consider one state per oscillator representing the position of the oscillator on its limit cycle. One requirement for the applicability of our method is that oscillators with different parameter sets need to have similar limit cycles, which might not hold true for all oscillators in a network when there is high cell-to-cell variability. Certain oscillators may have different qualitative dynamics, or even show no oscillations at all. At the onset of synchronization, the first oscillators to synchronize are those with similar dynamics and, by consequence, similar eigenfrequencies (Kuramoto 1984). It is a reasonable assumption that these are also the oscillators with parameter sets close to the nominal ones, such that their limit cycles fulfill the requirements above. Furthermore, due to the nonlinearity of oscillatory networks, it is not possible to provide an explicit relationship between the synchronization performance and, for instance, the steady-state distribution of the phases of the oscillators after synchronization. However, we argue that one can expect better synchronization results for an increasing value of the synchronization performance. Both considerations are confirmed by numerical simulations, which are in good agreement with the theoretical results and suggest a monotone relationship between synchronization performance and homogeneity of the phases of the oscillators after synchronization.

Finally, in recently published theoretical articles, we observed that the effect of the spatial distribution of the oscillators was often neglected by assuming the extracellular signaling molecules to be well mixed. We analyzed the effect of a spatial distribution of synchronized oscillating cells on a two-dimensional plate and explained the possibility of emerging phase waves similar to those recently observed experimentally by Danino et al. (2010) in vivo. We proposed an experimental setup with which 
the effect of these waves can be minimized and rapid synchronization can be achieved by the introduction of a non-homogeneous flow in the extracellular medium. We hope that our analysis method and results on the effect of cell density, parameter variability, and the coupling mechanism provide support for experimental biologists to successfully design and implement autonomously synchronizing oscillatory systems in vivo in the near future.

Acknowledgements We thank Prof. Stanly Steinberg for comments on the spatial diffusion model. This work was supported by the EC Framework 6 (COBIOS).

\section{References}

Acheson, D. J. (1990). Elementary fluid dynamics. London: Oxford University Press.

Atkinson, M. R., Savageau, M. A., Myers, J. T., \& Ninfa, A. J. (2003). Development of genetic circuitry exhibiting toggle switch or oscillatory behavior in Escherichia coli. Cell, 113, 597-607.

Brown, E., Moehlis, J., \& Holmes, P. (2004). On the phase reduction and response dynamics of neural oscillator populations. Neural Comput., 16, 673-715.

Daniels, B. C. (2005). Synchronization of globally coupled nonlinear oscillators: the rich behavior of the Kuramoto model. Technical report, Ohio Wesleyan University.

Danino, T., Mondragon-Palomino, O., Tsimring, L., \& Hasty, J. (2010). A synchronized quorum of genetic clocks. Nature, 463, 326-330.

Elowitz, M. B., \& Leibler, S. (2000). A synthetic oscillatory network of transcriptional regulators. Nature, $403,335-338$.

Ferziger, J. H., \& Peric, M. (1999). Computational methods for fluid dynamics (2nd ed.). Berlin: Springer.

Fung, E., Wong, W. W., Suen, J. K., Bulter, T., Lee, S., \& Liao, J. C. (2008). A synthetic gene-metabolic oscillator. Nature, 435, 118-122.

Garcia-Ojalvo, J., Elowitz, M. B., \& Strogatz, S. H. (2004). Modeling a synthetic multicellular clock: repressilators coupled by quorum sensing. Proc. Natl. Acad. Sci., 101(30), 10955-10960.

Gonze, D., Bernard, S., Waltermann, C., Kramer, A., \& Herzel, H. (2005). Spontaneous synchronization of coupled circadian oscillators. Biophys. J., 89, 120-129.

Goryachev, A. B., Toh, D. J., \& Lee, T. (2006). Systems analysis of a quorum sensing network: design constraints imposed by the functional requirements, network topology and kinetic constants. Biosystems, $83,178-187$.

Koseska, A., Volkov, E., Zaikin, A., \& Kurths, J. (2007a). Inherent multistability in arrays of autoinducer coupled genetic oscillators. Phys. Rev. E, 75, 031916-1-031916-8.

Koseska, A., Zaikin, A., Garcia-Ojalvo, J., \& Kurths, J. (2007b). Stochastic suppression of gene expression oscillators under intercell coupling. Phys. Rev. E, 75, 031917-1-031917-9.

Kuramoto, Y. (1984). Chemical oscillations, waves and turbulence. Berlin: Springer.

Kuramoto, Y., \& Nishikawa, I. (1987). Statistical macrodynamics of large dynamical systems. Case of a phase transition in oscillator communities. J. Stat. Phys., 49, 569-605.

Kuznetsov, A., Kaern, M., \& Kopell, N. (2004). Synchrony in a population of hysteresis-based genetic oscillators. SIAM J. Appl. Math., 65(2), 392-425.

Li, C., Chen, L., \& Aihara, K. (2007). Stochastic synchronization of genetic oscillator networks. BMC Systems Biology, 1(6).

Locke, J. C. W., Westermark, P. O., Kramer, A., \& Herzel, H. (2008). Global parameter search reveals design principles of the mammalian circadian clock. BMC Systems Biology, 2(22).

McMillen, D., Kopell, N., Hasty, J., \& Collins, J. J. (2002). Synchronizing genetic relaxation oscillators by intercell signaling. Proc. Natl. Acad. Sci., 99(2), 679-684.

Misra, J. C., \& Mitra, A. (2008). Synchronization among tumour-like cell aggregations coupled by quorum sensing: a theoretical study. Comput. Math. Appl., 55, 1842-1853.

Müller, J., Kuttler, C., Hense, B. A., Rothballer, M., \& Hartmann, A. (2006). Cell-cell communication by quorum sensing and dimension-reduction. J. Math. Biol., 53, 672-702.

Patankar, S. V., \& Spalding, D. B. (1971). A calculation procedure for heat, mass and momentum transfer in three-dimensional parabolic flows. Int. J. Heat Mass Transf., 15, 1787-1806. 
Preitner, N., Damiola, F., Lopez-Molina, L., Zakany, J., Duboule, D., Albrecht, U., \& Schibler, U. (2002). The orphan nuclear receptor REV-ERB $\alpha$ controls circadian transcription within the positive limb of the mammalian circadian oscillator. Cell, 110, 251-260.

Rosenblum, M., Pikovsky, A., Kurths, J., Schafer, C., \& Tass, P. A. (2001). Handbook of biological physics (Vol. 4, pp. 279-321). Amsterdam: Elsevier. Chapter 9.

Russo, G., \& di Bernardo, M. (2009). How to synchronize biological clocks. J. Comput. Biol., 16(2), 379-393.

Sewell, G. (2005). The numerical solution of ordinary and partial differential equations. New York: Wiley.

Shiner, E., Reddy, S., Timmons, C., Guigen, L., Williams, S., \& Rumbaugh, K. (2004). Construction of a bacterial autoinducer detection system in mammalian cells. Biol. Proced. Online, 6(1), 268-276.

Stricker, J., Cookson, S., Bennett, M. R., Mather, W. H., Tsimring, L. S., \& Hasty, J. (2008). A fast, robust and tunable synthetic gene oscillator. Nature, 456, 516-520.

Strogatz, S. H. (2000). From Kuramoto to Crawford: exploring the onset of synchronization in populations of coupled oscillators. Physica D, 143, 1-20.

Taylor, A. F., Tinsley, M. R., Wang, F., Huang, Z., \& Showalter, K. (2009). Dynamical quorum sensing and synchronization in large populations of chemical oscillators. Science, 323, 614-617.

Tigges, M., Marquez-Lago, T. T., Stelling, J., \& Fussenegger, M. (2009). A tunable synthetic mammalian oscillator. Nature, 457, 309-312.

Wang, W., \& Slotine, J. J. (2005). On partial contraction analysis for coupled nonlinear oscillators. Biol. Cybern., 92(1), 38-53.

Waters, C. M., \& Bassler, B. L. (2005). Quorum sensing: cell-to-cell communication in bacteria. Annu. Rev. Cell Dev. Biol., 21, 319-346.

Winfree, A. T. (1967). Biological rhythms and the behavior of populations of coupled oscillators. J. Theor. Biol., 16, 15-42.

Zhou, T., Zhang, J., Yuan, Z., \& Chen, L. (2008). Synchronization of genetic oscillators. Chaos, 18, 037126-1-037126-20. 